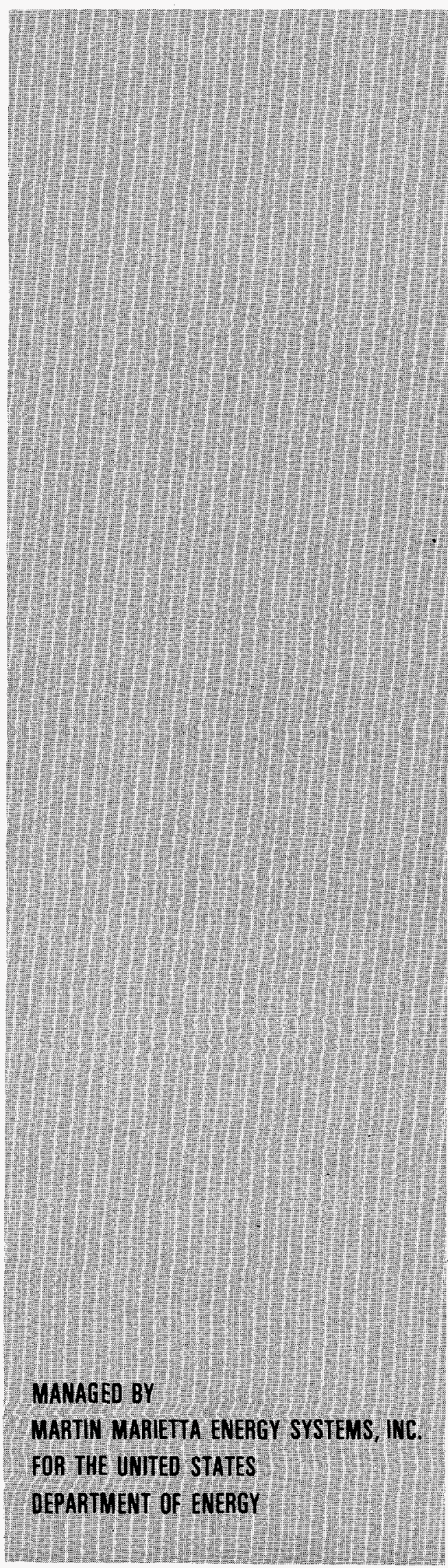

\author{
FLOOD ANALYSES FOR \\ DEPARTMENT OF ENERGY \\ Y-12, ORNL AND K-25 PLANTS
}

FLOOD ANALYSES IN SUPPORT OF FLOOD EMERGENCY PLANNING

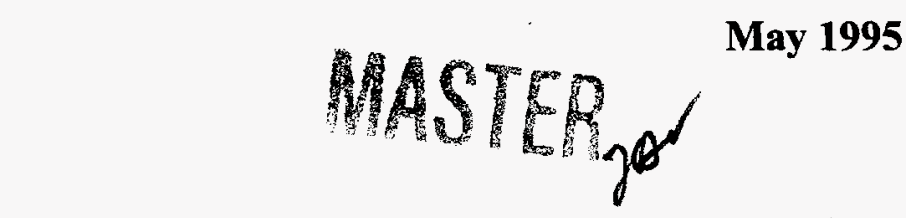

DISTRIBUTION OF THIS DOCUMENT IS UNLMTRED

Prepared for the Center for Natural Phenomena Engineering Oak Ridge Y-12 Plant Oak Ridge, TN 37831-8169 operated by Lockheed Martin Energy Systems, Inc. for the

U.S. Department of Energy under contract DE-AC05-84OR21400

RECEIVED AUG 281995

OSTI 


\author{
FLOOD ANALYSES FOR \\ DEPARTMENT OF ENERGY \\ Y-12, ORNL AND K-25 PLANTS \\ FLOOD ANALYSES \\ IN SUPPORT OF \\ FLOOD EMERGENCY PLANNING
}

December 1991

\author{
Prepared by \\ Flood Protection Section \\ Water Resources Operations Department \\ Water ResourcesDivision \\ Tennessee Valley Authority \\ Knoxville, TN 37902-1499
}

\title{
Prepared for the
}

Center for Natural Phenomena Engineering

Oak Ridge Y-12 Plant

Oak Ridge, TN 37831-8169

operated by

Lockheed Martin Energy Systems, Inc.

for the

U. S. Department of Energy

under contract $\mathrm{CE}$-AC05-84OR21400 


\section{DISCLAIMER}

Portions of this document may be illegible electronic image products. Images are produced from the best available original document. 
FLOOD ANALYSES

FOR

DEPARTMENT OF ENERGY

Y-12, ORNL, AND K-25 PLANTS

Submitted to:

Martin Marietta Energy Systems, Inc.

Interagency Agreement No. DE-AI05-910R21979

Flood Analyses in Support of Flood Emergency Planning

TVA Contract No. TV $-83730 \mathrm{~V}$

\author{
Submitted by: \\ Flood Protection Section \\ Water Resources Operations Department \\ Water Resources Division \\ Tennessee Valley Authority \\ Knoxville, Tennessee
}

December 1991 
I. GENERAL .......................... . . . . . . 1
A. Authority .................... . 1
B. Purpose .. . . . . . . . . . . . . . . 1
c. Scope ........................ 1

II. ENGINEERING METHODS . . . . . . . . . . . . . . 2

A. Hydrologic Analysis . . . . . . . . . . . . 2

1. TVA Maximum Probable and

Probable Maximum Flood Estimates . . . . . . 5

2. Precipitation Losses.............. . 5

B. Hydraulic Analysis . . . . . . . . . . . . . 7

C. Dam Failure Floods . . . . . . . . . . . . . . 9

III. RAINFALL DATA ... . . . . . . . . . . . . 10

Definition of Terms

\section{Figures}

1. Norris Dam - Assumed Condition of Dam After Failure

2. Norris Dam - Discharge Rating Curves for Failure Conditions

3. Melton Hill Dam - Discharge Rating Curve for Failure Condition

\section{Exhibits}

1. Clinch River, Elevations for Specified Events

2. East Fork Poplar Creek, Elevations for Specified Events

3. Poplar Creek, Elevations for Specified Events

4. Bear Creek, Elevations for Specified Events

5. Whiteoak Creek, Elevations for Specified Events

6. Melton Branch, Elevations for Specified Events

7. Local Area Rainfal1, Depth-Duration Data for 5-, 10-, 15-, and 30-Minute Rainfall

8. Local Area Rainfa11, Depth-Duration Data for 1-,2-,3-, and 6-Hour Rainfall

Appendix

References 
FOR

DEPARTMENT OF ENERGY

Y-12, ORNL, AND K-25 PLANTS

GENERAL

\section{Authority}

The work described in this report was conducted during June through November 1991 for Martin Marietta Energy Systems, Inc. (MMES) by the Tennessee Valley Authority (TVA) under the general supervision of Roger A. Milstead, Program Manager, Flood Hazard Analysis Unit, Flood Protection Section. The study was performed under Interagency Agreement No. DE-AI05-910R21979 - F1ood Analyses in Support of F1ood Emergency Planning - IVA Contract No. TV-83730V. A copy of this agreement is provided in the Appendix.

\section{Purpose}

The study involved defining the flood potential and local rainfall depth and duration data for the Department of Energy's (DOE) Y-12, Oak Ridge National Laboratory (ORNL), and $\mathrm{K}-25$ plants. All three plants are subject to flooding from the Clinch River. In addition, the $\mathrm{Y}-12$ plant is subject to flooding from East Fork Poplar and Bear Creeks, the ORNL plant from Whiteoak Creek and Melton Branch, and the $\mathrm{K}-25$ plant from Poplar Creek. Determination of flood levels included consideration of both rainfall events and postulated failures of Norris and Melton Hill Dams in seismic events.

Scope

The specific tasks called for providing the following information.

1. Clinch River flood elevations in the vicinity of the three plants and at the three DOE pumping stations located between Clinch River miles 11 and 12, 14 and 15 , and 41 and 42 for the following events.

A. The 25-, 100-, 500-, 2,000-, 10,000-, and 100,000-year, IVA maximum probable flood (TVA MPF), and probable maximum flood (PMF).

B. Postulated seismic failure of Norris Dam coincident with one-half PMF.

C. Postulated seismic failure of Norris Dam in nonflood conditions.

D. Postulated seismic failure of Melton Hill Dam coincident with one-half PMF.

E. Postulated seismic failure of Melton Hill Dam in nonflood conditions. 
2. East Fork Poplar Creek flood elevations for the 25-, 100-, 500-, 2,000-, 10,000-, and 100,000-year, TVA MPF, and PMF. Site drainage at Buildings 9201-2, 9201-3, 9204-1, 9720-1, and 9720-5.

3. Bear Creek flood elevations for the 25-, 100-, 500-, 2,000-, 10,000and 100,000-year, IVA MPF, and PMF.

4. Whiteoak Creek flood elevations for the 25-, 100-, 500-, 2,000-, $10,000-$, and 100,000-year, TVA MPF, and PMF.

5. Me1ton Branch flood elevations for the 25-, 100-, 500-, 2,000-, 10,000-, and 100,000-year, TVA MPF, and PMF.

6. Poplar Creek (to mile 5.63) flood elevations for the 25-, 100-, 500-, 2,000-, 10,000-, and 100,000-year, TVA MPF, and PMF.

7. Local rainfall depth and duration data for the 25-, 100-, 500-, 2,000-, 10,000-, and 100,000-year, TVA MPF, and PMF storm events.

\section{ENGINEER ING METHODS}

Standard hydrologic and hydraulic study methods were used to determine the flood potential for Poplar, East Fork Poplar, Bear, and Whiteoak Creeks, Melton Branch, and for floods up to the 500-year event on the Clinch River. Unsteady flow routing techniques (Reference 1) were used on the Clinch River to determine the TVA MPF and PMF elevations and flood elevations resulting from postulated failure of Norris and Melton Hill Dams.

\section{Hydrologic Analyses}

Hydrologic analyses were carried out to establish the peak dischargefrequency relationships for the six streams involved in this study. Discharge-frequency estimates for Poplar, Bear, and Whiteoak Creeks and Melton Branch were based on regional relationships of peak discharge and drainage area developed previously for flood studies made for the Federal Emergency Management Agency. These relationships were developed from analyses of records at the gaging stations shown in Table 1, Stream Gages, which are on the study streams and on nearby streams with similar hydrologic characteristics. Frequency curves for the gaged locations used to define the regional relationships were computed using the procedure outlined in U.S. Water Resources Council Bulletin 17B (Reference 2) and adjusted for the available historic flood information. The regional relationships were checked using updated frequency analysis of the gaging stations on the streams in the Oak Ridge area.

The adopted regional relationships were compared to the applicable USGS relationships for Tennessee (Reference 3 ). Discharge estimates from the adopted relationships ranged from 0 to 30 percent less than the USGS relationships for Tennessee, depending on the drainage area. The differences result mainly from the different gaged watersheds used in each analysis. The adopted regional relationships were developed from 
gages in the general vicinity of Oak Ridge. The USGS Tennessee relationships were developed using gages in the Cumberland Plateau, Valley and Ridge, and Blue Ridge physiographic provinces with varying hydrometeorologic characteristics.

\section{TABLE 1 - STREAM GAGES}

\section{Location}

Nails Creek near Knoxville Melton Branch near Oak Ridge Willow Fork near

Halls Crossroads

White Creek near Sharps Chapel

Whiteoak Creek below ORNL near Oak Ridge

Millican Creek near

Douglas Dam

Caney Creek near Kingston

Bear Creek near Oak Ridge

Buffalo Creek at Norris

Island Creek at Vonore

First Creek at Mineral Springs Ave

Big Barren Creek near New Tazewel1

Baker Creek near Greenback

First Creek at Fifth Avenue

East Fork Poplar Ck near Oak Ridge

Sweetwater Creek near Sweetwater

Bat. Creek near Vonore

Poplar Creek near Oliver Springs

Sweetwater Creek near Loudon

Bullrun Creek near

Halls Crossroads

West Prong Little Pigeon River near Pigeon Forge

Poplar Creek near Oak Ridge Little River near Maryville Little Pigeon River near Sevierville

\author{
Gage No/ \\ Agency
}

$03498700 /$ USGS

$03537500 /$ USGS

$03535180 /$ USGS

03528400/TVA

03537000/USGS

03469010/TVA

03538130/USGS

03538275/USGS

$03534500 /$ USGS

03519600/USGS

03496000/USGS

$03528300 /$ TVA

$03519640 /$ USGS

03496500/USGS

03538250/USGS

- ITVA

$03519700 /$ USGS

$03538200 /$ USGS

03520100/USGS

03535000/USGS

03469500/USGS

03538225/USGS

03498500/USGS

$03470000 /$ USGS
Drainage Area (Sq. Miles) of Record

$$
0.36
$$

1.48

1955-81

1956-64

2.67

2.68

1967-81

1937-72

3.62

1950-63

4.2

1942-48

1950-62

5.55

7.1

7.82

1962-81

1961-78

1948-50

1955-81

11.2

1954-76

11.9

1946-63

13.25

1935-45

1966-81

1946-58

16.6

1961-81

19.5

1965-78

28.1

1954-76

55.9

1954-81

62.2

1954-81

68.5

1958-81

76.2

1947-49

1954-81

82.5

1961-81

1951-81

353

1920-81 
For Bear Creek the principal source of flood information is records from USGS gage No. 03538275 at mile 0.9 (1961-78). Discharge-frequency at this gage computed using Bulletin 17B procedures (Reference 2) was considerably lower than that indicated by the regional relationships. Therefore, discharge-frequency estimates for Bear Creek were made by transfer using a flow-drainage area relationship through the gage point and parallel to the regional relationships. The upper reaches of Bear Creek are affected by urban development; the discharge-frequency estimates were increased to account for urban development using a procedure developed by the USGS (Reference 4). This procedure relates discharge for urban conditions to discharge for predevelopment conditions and watershed and climatic factors. The regional relationships adjusted to the Bear Creek gage were used to estimate the discharges for predevelopment conditions. Impervious areas and other urban data such as drainage improvement were provided by the Planning Office of the City of Oak Ridge. Channel slope, basin area, and storage were estimated from USGS 7.5-minute series topographic maps. Rainfall intensity was estimated using U.S. Weather Bureau Technical Paper No. 40 (Reference 5).

The principal source of flood information for Poplar Creek is records from USGS gage No. 03538225 at mile 13.8 (1961-89). The frequency curve for the gage station was computed using procedures outlined in Bulletin 17B (Reference 2). Discharge-frequency for the gage matched the regional relationships; therefore, discharge-frequency estimates for Poplar Creek were made using the regional relationships without adjustment.

Discharge frequency for Whiteoak Creek and Melton Branch was estimated using the regional relationships without adjustment. The dischargefrequency analysis for the USGS gage on Whiteoak Creek below ORNL (No. 03537000) was not used to adjust the regional relationships because of uncertainties with the gage discharge rating but was used as an indication that no urban adjustment is needed for the Whiteoak Creek or Melton Branch watersheds.

The principal source of flood information for East Fork Poplar Creek is records from USGS gage No. 03538250 at mile 3.3 (1961-88). A second USGS stream gage, No. 03538242 at mile 10.0 (1962-70), was not directly used because of the short period of record. Discharge-frequency at the gages was determined using the procedures outlined in Bulletin 17B (Reference 2).

East Fork Poplar Creek has been affected by significant urban development since Oak Ridge was established in 1942; however, most of the development on the East Fork Poplar Creek watershed occurred before the gage at mile 3.3 was installed. Stream gage records at mile 3.3 therefore reflect current urbanized conditions and were used to estimate urban dischargefrequency at the gage location. At locations above the stream gage where the watershed has a higher percent of development, estimates of urban discharge-frequency were made as follows. An estimate of discharge frequency at the gage for undeveloped conditions was made by subtracting from the gage discharge-frequency the effects of urbanization estimated using the USGS procedure (Reference 4) described above. Undeveloped 
discharge-frequency estimates for upstream locations were made by transfer using a flow-drainage area relationship through the undeveloped discharge-frequency estimate at the gage and parallel to the adopted regional relationships. The undeveloped discharge-frequency estimates were increased to account for urban development using the USGS procedure (Reference 4). Watershed and climatic factors were estimated as previously described.

Clinch River flows have been regulated by Norris Dam at mile 79.8 since closure in March 1936. Closure of Melton Hill Dam at mile 23.1 in May 1963 has not altered flood discharge frequency. Clinch River dischargefrequency relationships for the reaches above Enory River were determined from graphical analysis of Norris Dam discharges from 1937-1990, USGS gaging station records at Scarboro, mile 29, from 1937-1963, and Me1ton Hill Dam discharges from 1964-1990. For the 4.4-mile reach below Emory River maximum discharges results largely from the 865-square-mile Emory River watershed. Discharge-frequency for the reach below Emory River was computed using Bulletin 17B procedures (Reference 2) app1ied to maximum Emory River discharges plus concurrent Melton Hill Dam discharges.

TVA Maximum Probable Flood (IVA MPF) and Probable Maximun Flood (PMF) Estimates--Unit hydrographs were used to compute the TVA MPF and PMF. Unit hydrographs were developed for each study stream from maximum flood hydrographs recorded at stream gaging stations using the procedures described by Newton and Vinyard (Reference 6). Equations relating the unit graph peak flow to the drainage area size and time to peak in terms of watershed slope and length were developed from the computed unit graph parameters. These equations were used to develop synthetic unit graphs at ungaged locations.

The hydrologic runoff models used for the Clinch River and Tennessee River which produce controlling elevations near the mouth of the C1inch River are the models developed for TVA's nuclear plant siting studies and are described in the Watts Bar Nuclear Plant Final Safety Analysis Report (Reference 7).

TVA maximum probable precipitation (IVA MPP) and probable maximum precipitation (PMP) for the Clinch River and the other five study streams has been defined for TVA by Hydrometeorological Report No. 56 (Reference 8). Hydrometeorological Report No. 41 (Reference 9) defines TVA MPP and PMP for the Tennessee River watershed above Chattanooga. These reports define depth-area-duration characteristics and antecedent storm potentials. Table 2 provides TVA MPP and PMP estimates for the study streams and the Tennessee River.

Precipitation Losses-A multivariable relationship, used in the day-to-day operation of the TVA system, has been applied to determine precipitation excess $\mathrm{P}_{\mathrm{e}}$ directly. The relationships were developed from observed data and relate precipitation excess to the rainfall, week of the year, geographic location, and antecedent precipitation index (API). In their application $\mathrm{P}_{e}$ becomes an increasing fraction of rainfall as the storm progresses in time and becomes equal to rainfall when from 6 to 16 inches have fallen. For this study an API of 1.0 as determined from past studies was used at the start of the antecedent storm. 
TABLE 2 - TVA MAXIMUM PROBABLE AND PROBABLE MAXIMUM STORM RAINFALL

\section{Stream/Location}

Tennessee River above Watts

Bar Dam

Clinch River at Norris Dam at Melton Hill Dam

Poplar Creek at mouth

\section{E.F. Poplar Creek} at gage at mile 14.7

\section{Bear Creek} at mile 1.0 at mile 7.2

Whiteoak Creek at Whiteoak Dam at mile 4.0

\begin{tabular}{ll}
\multicolumn{2}{c}{ IVA Max Prob Rainfa11 } \\
\hline Antecedent & Main \\
Storm & Storm \\
Rain & Rain \\
(Inches) & (Inches)
\end{tabular}

4.2

10.5

6.8
Prob Max Rainfall

\begin{tabular}{ll}
\hline $\begin{array}{c}\text { Antecedent } \\
\text { Storm }\end{array}$ & $\begin{array}{c}\text { Main } \\
\text { Storm }\end{array}$ \\
$\begin{array}{c}\text { Rain } \\
\text { (Inches) }\end{array}$ & Rain \\
(Inches)
\end{tabular}

1.7

1.6

3.2

0.5

0.5

0.5

0.5

22.6

23.1

0.5

0.5
21.5

20.8

22.9

11.2

10.4

5.5

5.1

10.7

4.3

4.8

36.1

39.7

18.4

17.1

35.6

17.2

4.7

39.1

4.8

40.1

Tennessee River - TVA MPP occurs over a 10-day period: 3-day antecedent storm, 4-day dry period, and 3-day main storm. PMP occurs over 9-days: 3-day antecedent storm, 3-day dry period, and 3-day main storm. Antecedent rain equals 40 percent of main storm rain.

C1inch River

- Both events occur over a 9-day period: 3-day antePoplar Creek cedent storm, 3-day dry period, and 3-day main storm. Antecedent rain in TVA MPP is 15 percent of main storm rain; in PMP, 30 percent.

E.F. Poplar Ck Bear Creek Whiteoak Creek Melton Branch
- Both events result from 3-day storms: 2-day storm antecedent to the 1 -day main storm. Antecedent rain in TVA MPP is 0.25 inch per day for the 2-day antecedent event; in the PMP, antecedent rain is 2 percent of main storm rain on first day and 10 percent on second day. 
For review purposes, precipitation losses have been determined by subtracting $\mathrm{P}_{e}$ from rainfall. For example, in the probable maximum storm on East Fork Poplar Creek at the gage, losses are 5.53 inches, amounting to 14 percent of the 3 -day rainfall. In the probable maximum storm on Poplar Creek, losses are 5.48 inches, amounting to 51 percent of rainfal1, for the 3-day antecedent storm and 2.89 inches, 8 percent of rainfal1, for the 3-day main storm.

\section{Hydraulic Analyses}

Analyses of the hydraulic characteristics of flooding from the streams studied were carried out to provide estimates of the elevations of floods for the selected recurrence intervals.

Water-surface elevations for the 25-, 100-, and 500-year flood elevations for the Clinch River and for the 25-, 100-, and 500-year, TVA MPF, and PMF elevations for the other streams were computed using the U.S. Army Corps of Engineers (USACE) HEC-2 standard step-backwater computer program (Reference 10). Flood elevations for the 2,000-, 10,000-, and 100,000-year floods were estimated from elevation-frequency curves plotted on Gumbel frequency distribution paper, with the PMF plotted at a probability of $1 \times 10^{-6}$ and the TVA MPF plotted at a probability of $5 \times 10^{-5}$ (for basis, see text on rainfall data, page 10 of this report).

Elevations for the Tennessee River at the mouth of the Clinch River were taken from previous studies made for Watts Bar Reservoir.

Cross sections for the USACE HEC-2 model used for the Clinch River were based on silt range surveys and detailed topographic maps. The model was calibrated using March 1973 floodmarks and floodflows. Controlling flood elevations on the lower reaches of the Clinch River result from Tennessee River backwater.

The USACE HEC-2 model used for East Fork Poplar Creek included cross section data furnished by MMES and cross section data surveyed by TVA outside DOE property. The model was calibrated using the January 1982 and November 1977 floodmarks. The flood computations were made assuming that the inlets to Lake Reality were closed and that flow would be in the diversion channel and over Lake Reality embankments. Controlling flood elevations on the lower reaches of East Fork Poplar Creek result from Poplar Creek backwater.

Cross sections for the Poplar Creek model were furnished by MMES and the model was calibrated using the April 1977 flood. The elevations on the lower reaches of Poplar Creek result from Clinch and Tennessee River backwater.

Natural ground cross-sectional data for the Bear Creek model were furnished by MMES and the bridge sections were field surveyed by TVA. Gage data at miles $2.3,2.8$, and 3.9 for the January 1988 flood were used to calibrate the model. The elevations on the lower reaches of Bear Creek result from Poplar and East Fork Poplar Creeks backwater. 
Cross sections used for the Whiteoak Creek USACE HEC-2 model were furnished by MMES. The model used for this study was one previously developed, modified by adding additional cross sections. Whiteoak Dam headwater elevations defined in $\mathrm{Dr}$. B. A. Tschantz's report entitled "Whiteoak Creek Hydrologic and Spillway Adequacy Analysis" (Reference 11) were used in the analysis. Controlling flood elevations on the lower reaches of Whiteoak Creek result from Clinch and Tennessee River backwater.

Melton Branch and the tributary to Melton Branch cross-sectional data for the USACE HEC-2 model were furnished by MMES. Gage data for the January 1988 flood at the gage on Melton Branch at mile 1.2 were used to check the model. Backwater from the Clinch and Tennessee Rivers and Whiteoak Creek produce maximum flood elevations on the lower reaches of Melton Branch.

Unsteady flow techniques (Reference 1) were used on the Clinch River to determine TVA MPF and PMF elevations. The unsteady flow mathematical model for Clinch River was developed earlier in connection with TVA's nuclear plant siting studies and is described in the Clinch River Breeder Reactor Plant Preliminary Safety Analysis Report (Reference 12). Norris and Melton Hill Dams can safely pass the PMF (except for Melton Hill where the short section of the earth embankment section at the left abutment could possibly fail, which would not significantly increase discharges) and TVA judges both dams to be structurally competent.

Unsteady flow techniques (Reference 1) were also used on the Tennessee River to determine Tennessee River TVA MPF and PMF elevations (elevations from the Tennessee River are controlling on the lower reaches of the Clinch River). The unsteady flow mathematical model for the Tennessee River, developed for TVA's nuclear plant studies, is described in the Watts Bar Nuclear Plant Final Safety Analysis Report (Reference 7). Tennessee River elevations reflect the recently completed dam safety modification at Fort Loudoun-Tellico and the proposed dam safety modification at Watts Bar Dam, which calls for raising the embankment 10 feet; these modifications enable these projects to safely pass the PMF.

Elevations for the specified recurrence intervals for the six streams studied are shown in Exhibits 1 through 6. Maximum flood elevations at Y-12 Plant Buildings 9201-2, 9201-3, 9204-1, 9720-1, and 9720-5 would result from the PMF on East Fork Poplar Creek. Table 3 provides PMF elevations, together with the TVA MPF elevations, at these five buildings. 
TABLE 3 - PMF AND TVA MPF ELEVATIONS

AT SPECIFIED Y-12 BUILDINGS

\section{Building/Location}

9201-3/E.F. Poplar Cr. mile 15.34

(upstream side of building)

9201-2/E.F. Poplar Cr. mile 15.45

(center of building)

9720-1/E.F. Poplar Cr. mile 15.70

(upstream side of building)

9204-1/E.F. Poplar Cr. mile 15.78

(center of building)

9720-5/E.F. Poplar Cr. mile 16.28

(downstream side of building;

at entrance to long underground culvert)

\begin{tabular}{ll}
\multicolumn{2}{c}{ Elevation } \\
\hline TVA MPF & $\underline{\text { PMF }}$ \\
929.6 & 933.0 \\
931.2 & 935.0 \\
941.6 & 948.0 \\
942.5 & 949.0 \\
968.0 & 969.6
\end{tabular}

\section{Dam Failure Floods}

Norris and Melton Hill Dams (separately) were postulated to fail seismically concurrent with the one-half $\mathrm{PMF}$ and in nonflood conditions. Figure 1 shows the 665-foot-long section of Norris Dam postulated to fail. The procedures and specific analysis to determine Norris Dam stability are described in Reference 12. Discharge rating curves for Norris Dam for two different failure conditions are shown on Figure 2; the rating used for this study was that for the 665 feet of overturned dam. These rating curves were developed from 1:150-scale hydraulic model studies at TVA's Engineering Laboratory and verified closely by hydraulic analysis.

The mode of failure postulated for Melton Hill Dam included failure of the left nonoverflow portion of the dam to elevation 774.5 and the left abutment to rock at about elevation 760 . The rating curve for Melton Hill Dam for failure conditions is shown on Figure 3.

By furnishing this information TVA neither implies nor concedes that its dams are inadequate to withstand great floods and/or earthquakes that may be reasonably expected to occur in the TVA region under consideration. TVA has a program of inspection and maintenance carried out on a regular schedule to keep its dams safe. Instrumentation of the dams to help keep check on their behavior was installed in many of the dams during original construction. Other instrumentation has been added since and is still being added as the need may appear or as new techniques become available. In short, TVA has confidence that its dams are safe against catastrophic destruction by any natural forces that could be expected to occur.

Failure of Norris and Melton Hill Dams during one-half the PMF was assumed 
to occur at peak reservoir levels; at Norris, this elevation was 1036.9 and at Melton Hill, 799.3. Reservoir levels for the nonflood failure were assumed at normal maximum pool elevation 1020 for Norris and 795 for Melton Hill. Failure of Norris Dam in both events would overtop and fail Melton Hill Dam. Unsteady flow techniques (Reference 1) were used to route the floods resulting from the dam failures. Exhibit 1 provides elevations for these postulated failure conditions.

\section{RAINFALL DATA}

Rainfall frequency curves were developed for local area rainfall using estimates of the 5-, 10-, 25-, 50-, and 100-year rainfall and the IVA maximum probable precipitation (TVA MPP) and probable maximum precipitation (PMP). Order-of-magnitude estimates of the probability of the IVA MPP and PMP were made based on extrapolating flood data, watershed rainfall, and extraordinary storm occurrences.

Rainfal1-frequency estimates for durations from 5 to 60 minutes and return periods up to 100 years were obtained from Nationa1 Oceanic and Atmospheric Administration's (NOAA) Technical Memorandum NWS HYDRO-35 (Reference 13). Estimates for durations longer than 1 hour and return periods up to 100 years were obtained from U.S. Weather Bureau's Technical Paper No. 40 (Reference 5). TVA MPP and PMP estimates were obtained from Hydrometeorologic Report No. 56 (Reference 8).

To establish the exceedance probability of the TVA MPP, earlier estimates of flood-frequency curves at 36 long-record stream gaging stations extrapolated to computed IVA maximum probable flood (IVA MPF) estimates were reviewed. The 36 watersheds were within the Tennessee Valley watershed and ranged from 31.9 square miles to 21,400 square miles. The TVA MPF exceedance probabilities ranged from $1 \times 10^{-3}$ to $10^{-9}$ with a median and mode of $1 \times 10^{-5}$. The exceedance probability of the IVA MPP was assumed equal to that of the TVA MPF.

Earlier estimates of the exceedance probability of the PMF (References 14 and 15) were reviewed; in particular, estimates based upon two rainfallfrequency analyses which considered (1) rainfall on watersheds within the Tennessee Valley and (2) storms occurring east of the 105 th meridian.

The exceedance probability of the PMF can be assumed equal to the exceedance probability of the PMP; this is because the PMP is defined as an event approaching the physical upper limit of precipitation.

Rainfal1-frequency curves of maximum annual 3-day watershed storm rainfall were computed for five watersheds in the Tennessee Valley ranging in area from 232 square miles to 20,000 square miles. Extrapolation of these curves to PMP estimates developed by the National Weather Service (References 9 and 16) indicated that exceedance probabilities range from $3 \times 10^{-8}$ to $1 \times 10^{-17}$. 
To determine the chance of a PMP storm striking a selected area, observed 6- and 24-hour rainfalls for storms covering 10 square miles and for 72-hour rainfalls covering 5,000 square miles east of the 105 th meridian greater than or equal to 50 percent of the PMP (Reference 17) were evaluated. These areas approximate the watershed sizes of the DOE study. Although no PMP storms have occurred, data are available from storms that were from 50 to 90 percent of the PMP storm and struck storm areas of 10 and 5,000 square miles. Extrapolation of these data to the PMP indicate that exceedance probabilities range from $2.4 \times 10^{-7}$ to $5.6 \times 10^{-8}$.

Based on this information, an exceedance probability of $1 \times 10^{-8}$ was assumed for the PMP.

Determination of confidence intervals for the rainfall-frequency curves requires knowledge of the population distribution. The population distribution was assumed to be the Fisher-Tippett type I distribution with application as described by Gumbel (Reference 18), herein referred to as the Gumbel distribution. This is consistent with NOAA procedures which use the Gumbel frequency distribution. A least-squares regression analysis was used to fit the Gumbel distribution to the sample points for the 5-minute and 1-hour rainfall. A Smirnov-Kolmogorov ( $S-K$ ) goodnessof-fit test accepted the hypothesis that the sample points were from the Gumbel distribution. However, the $S-K$ goodness-of-fit test is not robust at small exceedance probabilities. Therefore, in order to be conservative, the upper 99 percent confidence interval $\left(10^{-6}\right)$ was adopted as the exceedance probability of the PMP. This is consistent with the American Nuclear Society's ANSI/ANS 2.8, 1981 guidelines (Reference 19), which state that "an average annual exceedance probability less than $10^{-6}$ is an acceptable goal for selection of flood design bases for power reactor plants" and accepts precipitation floods resulting from PMP as adequate design flood bases. Extrapolation of the rainfal1-frequency curves to the PMP with a probability of $1 \times 10^{-6}$ results in an exceedance probability of $5 \times 10^{-5}$ for the TVA MPP. Exhibits 7 and 8 provide local area rainfall depth-duration-frequency data. 


\section{Probable Maximum Precipitation (PMP)}

PMP is defined as "the theoretically greatest depth of precipitation for a given duration that is physically possible over a particular drainage area at a certain time of year" (American Meteorological Society, 1959). In consideration of our limited knowledge of the complicated processes and interrelationships in storms, PMP values are identified as estimates.

\section{Probable Maximum Flood (PMF)}

The most severe flood that can reasonably be predicted to occur at a site as a result of hydrometeorological conditions. It assumes an occurrence of PMP critically centered on the watershed and a sequence of related meteorologic and hydrologic factors typical of extreme storms.

\section{IVA Maximum Probable Flood (IVA MPF)}

A flood which is comparable to all but the more extreme floods known to have occurred in the eastern part of the United States. The rainfall used to estimate the flood (National Weather Service Hydrometeorological Reports 41, 47, and 56) was determined by transposing to the Tennessee Valley without maximization all but the most extreme storms which have occurred elsewhere in the eastern United States and is approximately 60 percent of the PMP. The resulting floods equal about 40 to 60 percent of the PMF for the same drainage basin.

\section{5-Year Flood}

The flood with a 1 in 25 ( 4 percent) chance of being equaled or exceeded in any given year.

\section{0-Year F1ood}

The flood with a 1 in 100 ( 1 percent) chance of being equaled or exceeded in any given year.

\section{0-Year F10od}

The flood with a 1 in 500 ( 0.2 percent) chance of being equaled or exceeded in any given year.

\section{2,000-Year Flood}

The flood with a 1 in 2,000 (.05 percent) chance of being equaled or exceeded in any given year.

\section{0,000-Year Flood}

The flood with a 1 in 10,000 (.01 percent) chance of being equaled or exceeded in any given year.

\section{0,000-Year Flood}

The flood with a 1 in 100,000 (.001 percent) chance of being equaled or exceeded in any given year. 

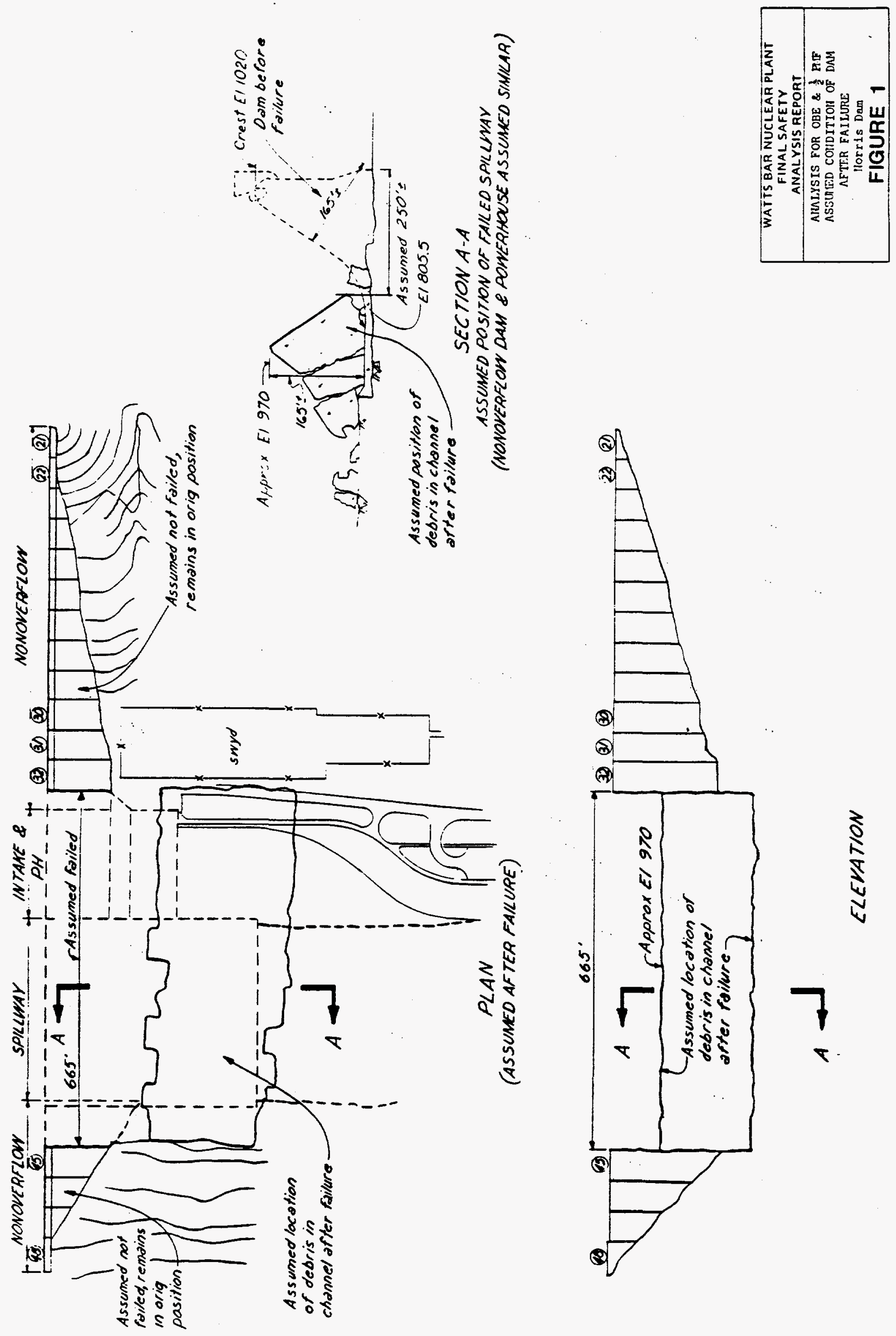
FIGURE 2

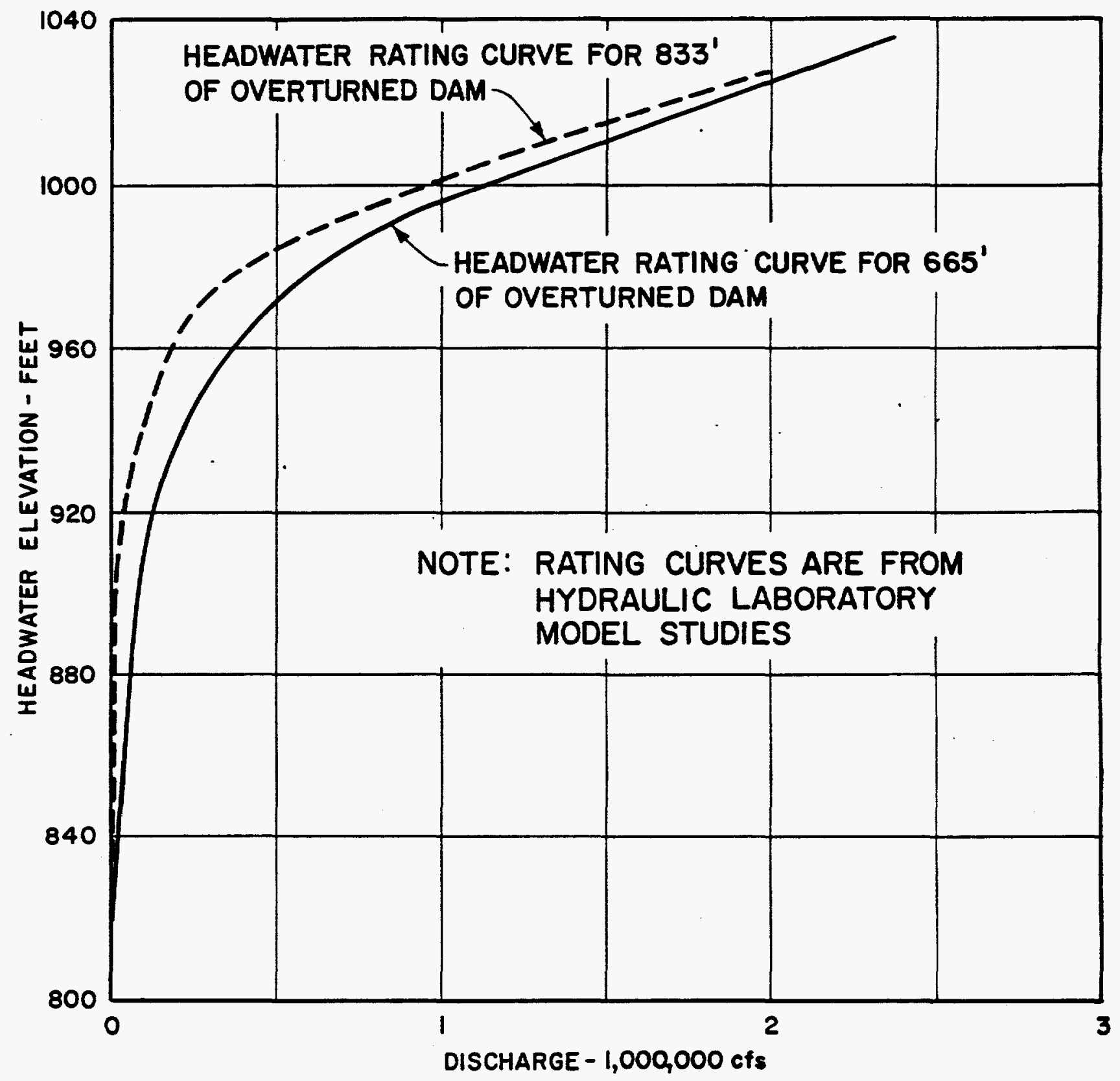

HEADWATER RATING CURVES

NORRIS DAM 


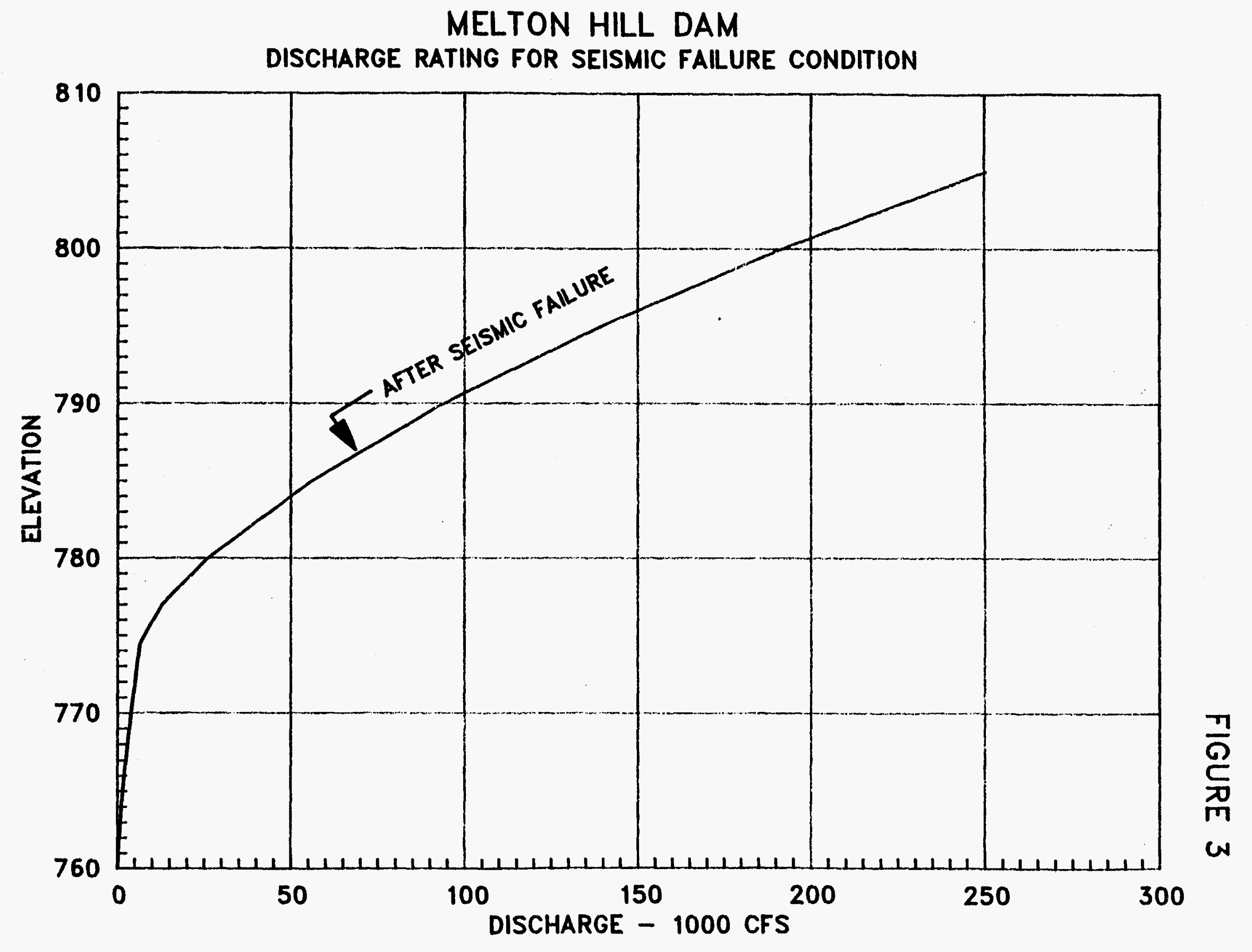


Elevations for Specified Events

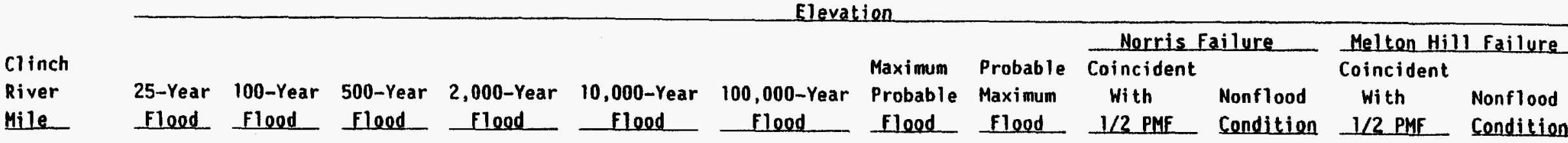

\section{K-25 SITE}

\begin{tabular}{|c|c|c|c|c|c|c|c|c|c|c|c|c|}
\hline 0.00 & $746.0^{*}$ & $747.1^{*}$ & $748.4^{*}$ & $751.2^{\star}$ & $756.2^{*}$ & $766.2^{\star}$ & $759.0^{*}$ & $776.7^{\star}$ & 760.0 & 754.6 & 750.1 & 742.4 \\
\hline 2.10 & $746.0^{\star}$ & $747.1^{*}$ & $748.4^{*}$ & $751.2^{\star}$ & $756.2^{\star}$ & $766.2^{\star}$ & $759.0^{*}$ & $776.7^{\star}$ & 760.2 & 755.2 & 750.2 & 742.4 \\
\hline 3.05 & $746.0^{*}$ & $747.1^{*}$ & $748.4^{*}$ & 751.6 & $756.3^{*}$ & $766.2^{\star}$ & $759.0^{*}$ & $776.7^{\star}$ & 763.0 & 757.3 & 750.7 & 742.4 \\
\hline 4.20 & $746.0^{*}$ & 747.6 & 749.4 & 752.1 & $756.5^{*}$ & $766.2^{\star}$ & $759.0^{*}$ & $776.7^{\star}$ & 766.3 & 759.9 & 751.4 & 742.5 \\
\hline 6.30 & $746.0^{\star}$ & 747.7 & 749.6 & 752.2 & $756.5^{*}$ & $766.3^{\star}$ & $759.1^{\star}$ & $776.8^{\star}$ & 768.7 & 762.6 & 752.4 & 742.6 \\
\hline 8.40 & $746.0 *$ & 748.0 & 750.1 & 752.7 & $756.8^{*}$ & $766.3^{\star}$ & $759.1^{\star}$ & $776.9^{\star}$ & 776.4 & 768.9 & 753.4 & 743.3 \\
\hline 10.50 & 746.3 & 748.7 & 751.1 & 753.5 & $757.1^{*}$ & $766.5^{\star}$ & $759.3^{\star}$ & $777.2^{\star}$ & 784.7 & 776.3 & 755.4 & 744.6 \\
\hline 12.60 & 746.5 & 749.0 & 751.6 & 754.0 & $757.4^{\star}$ & $766.7^{\star}$ & $759.4^{*}$ & $777.4^{\star}$ & 789.0 & 780.2 & 756.7 & 745.2 \\
\hline 14.70 & 747.2 & 750.0 & 753.0 & 755.4 & $758.0^{*}$ & $767.0^{\star}$ & $759.6^{\star}$ & $777.6^{\star}$ & 791.3 & 782.9 & 758.1 & 746.8 \\
\hline 16.80 & 747.9 & 751.0 & 754.2 & 756.5 & $758 . B^{*}$ & $767.2^{\star}$ & $759.8^{\star}$ & $778.0^{\star}$ & 797.3 & 788.2 & 759.7 & 748.5 \\
\hline
\end{tabular}

ORML SITE

\begin{tabular}{|c|c|c|c|c|c|c|c|c|c|c|c|c|}
\hline 18.90 & 749.2 & 752.8 & 756.5 & 758.3 & 759.9 & $767.5^{\star}$ & 761.0 & $778.7^{\star}$ & 804.4 & 794.6 & 763.1 & 752.6 \\
\hline 21.00 & 750.2 & 754.0 & 757.9 & 760.0 & 762.4 & $768.8^{*}$ & 763.5 & $779.4^{\star}$ & 810.5 & 799.4 & 765.7 & 755.4 \\
\hline (MH) 23.10 (TW) & 751.4 & 755.3 & 759.2 & 761.9 & 764.9 & $771.0^{\star}$ & 766.2 & 779.9* & 813.8 & 800.8 & 767.9 & 759.0 \\
\hline (MH) 23.10 (HW) & 796.0 & 796.0 & 796.0 & 796.0 & 797.7 & 803.2 & 799.3 & 808.8 & 831.2 & 818.8 & - & - \\
\hline 27.46 & 796.1 & 796.1 & 796.2 & 796.3 & 798.0 & 803.7 & 799.7 & 809.4 & 834.0 & 822.8 & - & - \\
\hline 31.82 & 796.1 & 796.2 & 796.4 & 796.5 & 798.3 & 804.2 & 800.0 & 810.0 & 837.0 & 827.0 & - & - \\
\hline \multicolumn{13}{|l|}{ Y-12.SIIE } \\
\hline 36.18 & 796.2 & 796.3 & 796.6 & 796.8 & 798.7 & 804.5 & 800.4 & 810.5 & 840.0 & 829.0 & - & - \\
\hline 40.55 & 796.3 & 796.7 & 797.1 & 797.4 & 799.3 & 805.3 & 801.0 & 811.6 & 844.0 & 834.0 & - & - \\
\hline 44.91 & 796.5 & 797.0 & 797.7 & 798.2 & 800.2 & 806.7 & 801.9 & 813.4 & 848.8 & 838.0 & - & - \\
\hline 49.27 & 796.8 & 797.5 & 798.3 & 799.0 & 801.0 & 807.9 & 802.7 & 815.2 & 851.2 & 843.0 & - & - \\
\hline 53.63 & 797.5 & 798.4 & 799.5 & 800.5 & 802.3 & 809.4 & 804.1 & 817.0 & 855.7 & 845.6 & - & - \\
\hline
\end{tabular}

"Controlling elevations are from the Tennessee River. Tennessee River elevations reflect the recently completed dam safety modification at

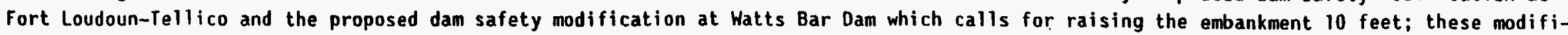
cations enable these projects to safely pass the PMF. 
EAST FORK POPLAR CREEK

Elevations for Specified Events

\begin{tabular}{|c|c|c|c|c|c|c|c|c|}
\hline & & & & & & & & \\
\hline E.F. & & & & & & & Maximum & Probable \\
\hline Poplar & $25-Y r$ & $100-Y r$ & $500-Y r$ & $2,000-Y r$ & $10,000-Y r$ & $100,000-\gamma_{r}$ & Probable & Maximum \\
\hline Creek & Flood & Flood & Flood & Flood & Flood & Flood & Flood & Flood \\
\hline $0.04 \mathrm{DS}$ & $754.6^{\star}$ & $758.2^{*}$ & $762.2^{\star}$ & $764.5^{\star}$ & $767.3^{\star}$ & $772.5^{*}$ & $768.5^{\star}$ & $778.9^{*}$ \\
\hline 0.04 us & $754.6^{*}$ & $758.2^{\star}$ & $762.2^{\star}$ & $764.5^{\star}$ & $767.3^{\star}$ & $772.5^{\star}$ & $768.5^{\star}$ & $778.9 *$ \\
\hline 0.74 & $754.6^{x}$ & $758.2^{\star}$ & $762.2^{n}$ & $764.5^{\star}$ & $767.3^{\star}$ & $772.5^{\star}$ & $768.5^{*}$ & $778.9^{*}$ \\
\hline 1.20 & 755.6 & $758.2^{\star}$ & $762.2^{\star}$ & $764.5^{\star}$ & $767.3^{\star}$ & $772.5^{*}$ & $768.5^{\star}$ & $778.9^{*}$ \\
\hline 1.40 & 755.9 & $758.2^{\star}$ & $762.2^{\star}$ & $764.5^{\star}$ & $767.3^{\star}$ & $772.5^{\star}$ & $768.5^{\star}$ & $778.9^{*}$ \\
\hline 1.63 & 756.3 & 758.6 & $7.62 .2^{\star}$ & $764.5^{\star}$ & $767.3^{\star}$ & $772.5^{\star}$ & $768.5^{\star}$ & $778.8^{\star}$ \\
\hline 1.66 & 756.4 & 758.7 & $762.2^{*}$ & $764.5^{*}$ & $767.3^{*}$ & $772.5^{\star}$ & 768.6 & $778.9 *$ \\
\hline 1.92 & 757.2 & 759.4 & $762.2^{\star}$ & $764.5^{\star}$ & 767.8 & $772.5^{\star}$ & 769.1 & $778.9^{x}$ \\
\hline 2.14 & 757.8 & 759.9 & $762.2^{\star}$ & $764.5^{\star}$ & 768.2 & 773.2 & 769.6 & $778.9^{\star}$ \\
\hline 2.20 & 758.0 & 760.1 & 762.3 & $764.5^{\star}$ & 768.3 & 773.3 & 769.7 & $778.9^{*}$ \\
\hline 2.36 & 758.5 & 760.5 & 762.7 & 765.2 & 768.6 & 773.8 & 770.1 & $778.9^{*}$ \\
\hline 2.94 & 763.2 & 764.9 & 766.8 & 769.2 & 772.0 & 775.7 & 773.3 & $778.9^{*}$ \\
\hline 3.00 & 763.7 & 765.4 & 767.2 & 769.6 & 772.4 & 775.9 & 773.6 & 779.3 \\
\hline $3.32 \mathrm{dS}$ & 768.9 & 771.0 & 772.8 & 774.7 & 777.0 & 780.3 & 778.0 & 783.7 \\
\hline 3.32 US & 769.5 & 771.2 & 773.0 & 774.9 & 777.1 & 780.4 & 778.1 & 783.7 \\
\hline 3.79 & 772.2 & 773.8 & 775.6 & 777.4 & 779.7 & 783.2 & 780.7 & 786.8 \\
\hline 3.88 & 772.5 & 774.1 & 775.9 & 777.7 & 779.9 & 783.4 & 781.0 & 786.8 \\
\hline 3.96 & 773.2 & 774.8 & 776.6 & 778.3 & 780.7 & 784.4 & 781.8 & 788.2 \\
\hline 4.59 & 777.3 & 778.9 & 780.7 & 782.4 & 784.8 & 788.5 & 785.9 & 792.3 \\
\hline 4.78 DS & 778.6 & 780.2 & 782.0 & 783.8 & 786.1 & 789.6 & 787.2 & 793.5 \\
\hline 4.78 US & 779.4 & 781.9 & 783.2 & 784.5 & 786.5 & 790.0 & 787.5 & 793.6 \\
\hline 4.93 & 780.2 & 782.4 & 783.7 & 785.2 & 787.2 & 790.7 & 788.2 & 794.3 \\
\hline 5.12 & 781.1 & 783.1 & 784.5 & 786.0 & 788.0 & 791.3 & 789.0 & 795.1 \\
\hline 5.74 & 786.2 & 787.6 & 789.2 & 791.1 & 793.3 & 796.7 & 794.3 & 800.1 \\
\hline 6.17 & 790.1 & 791.5 & 793.1 & 795.0 & 797.3 & 800.6 & 798.3 & 803.9 \\
\hline 6.22 & 790.5 & 792.0 & 793.6 & 795.5 & 797.8 & 801.1 & 798.8 & 804.4 \\
\hline 6.72 & 793.2 & 794.5 & 796.0 & 797.8 & 800.1 & 803.3 & 801.2 & 807.1 \\
\hline 6.80 & 793.7 & 794.9 & 796.3 & 798.3 & 800.5 & 803.7 & 801.5 & 807.3 \\
\hline $6.89 \mathrm{DS}$ & 794.6 & 795.6 & 796.9 & 798.8 & 800.9 & 803.9 & 801.8 & 807.6 \\
\hline 6.89 US & 796.3 & 797.5 & 798.5 & 799.8 & 801.4 & 804.1 & 802.1 & 807.8 \\
\hline 7.15 & 798.5 & 799.8 & 801.1 & 802.8 & 804.8 & 807.6 & 805.6 & 810.7 \\
\hline 7.35 & 802.1 & 803.6 & 805.4 & 807.5 & 809.9 & 813.4 & 811.0 & 816.7 \\
\hline 7.85 & 808.2 & 809.9 & 811.8 & 814.2 & 816.8 & 820.6 & 818.0 & 824.4 \\
\hline $7.95 \mathrm{DS}$ & 809.2 & 810.9 & 812.9 & 815.2 & 817.8 & 821.6 & 819.0 & 825.4 \\
\hline 7.95 US & 809.3 & 811.1 & 813.1 & 815.4 & 818.1 & 821.8 & 819.2 & 825.6 \\
\hline 8.30 & 811.5 & 813.4 & 815.6 & 818.1 & 821.1 & 825.1 & 822.4 & 829.1 \\
\hline 8.39 & 812.0 & 814.0 & 816.3 & 819.0 & 822.2 & 826.4 & 823.5 & 830.8 \\
\hline $8.45 \mathrm{DS}$ & 812.4 & 814.3 & 816.5 & 819.2 & 822.4 & 826.7 & 823.7 & 831.0 \\
\hline 8.45 US & 813.4 & 815.4 & 818.0 & 822.1 & 825.7 & 829.7 & 826.9 & 833.3 \\
\hline 8.70 & 814.4 & 816.2 & 818.6 & 822.5 & 826.0 & 830.3 & 827.4 & 834.1 \\
\hline 8.80 & 814.9 & 816.5 & 818.8 & 822.6 & 826.1 & 830.4 & 827.5 & 834.2 \\
\hline
\end{tabular}

\footnotetext{
* Controlling elevations are from Poplar Creek
} 
EAST FORK POPLAR CREEK

Elevations for Specified Events (Continued)

\begin{tabular}{|c|c|c|c|c|c|c|c|c|}
\hline \multirow{2}{*}{ E.F. } & \multicolumn{8}{|c|}{ Elevation } \\
\hline & & & & & & & \multirow{3}{*}{$\begin{array}{l}\text { Maximum } \\
\text { Probable } \\
\text { Flood } \\
\end{array}$} & \multirow{3}{*}{$\begin{array}{l}\text { Probable } \\
\text { Maximum } \\
\text { Flood } \\
\end{array}$} \\
\hline $\begin{array}{l}\text { E.F. } \\
\text { Poplar }\end{array}$ & $25-Y r$ & $100-Y r$ & $500-Y r$ & $2,000-Y r$ & $10,000-Y r$ & $100,000-Y r$ & & \\
\hline Creek & Flood & Flood & Flood & Flood & Flood & Flood & & \\
\hline $9.00 \mathrm{DS}$ & 816.7 & 817.8 & 819.6 & 823.1 & 826.4 & 830.6 & 827.7 & 834.4 \\
\hline 9.00 US & 818.0 & 819.2 & 820.6 & 823.1 & 826.4 & 830.6 & 827.7 & 834.4 \\
\hline 9.21 & 819.4 & 820.7 & 822.2 & 824.7 & 827.5 & 831.3 & 828.6 & 835.1 \\
\hline 9.45 DS & 820.5 & 821.7 & 823.1 & 825.4 & 828.0 & 831.8 & 829.2 & 835.6 \\
\hline 9.45 US & 821.9 & 822.6 & 823.4 & 825.4 & 828.0 & 831.8 & 829.2 & 835.6 \\
\hline 9.55 & 822.2 & 823.0 & 823.9 & 826.0 & 828.4 & 832.1 & 829.5 & 835.9 \\
\hline 9.74 & 823.8 & 824.6 & 825.7 & 827.2 & 829.5 & 833.1 & 830.7 & 836.8 \\
\hline $10.00 \mathrm{DS}$ & 827.4 & 827.9 & 828.9 & 830.0 & 831.7 & 834.8 & 832.7 & 838.1 \\
\hline 10.00 US & 830.6 & 831.7 & 833.2 & 833.9 & 835.1 & 836.9 & 835.7 & 838.6 \\
\hline 10.15 & $831 \ldots 0$ & 832.0 & 833.6 & 834.7 & 835.9 & 837.8 & 836.5 & 839.7 \\
\hline 10.53 & 855.2 & 836.0 & 637.3 & 856.7 & 840.7 & 943.2 & 841.5 & 845.5 \\
\hline 11.10 & 839.1 & 840.0 & 841.3 & 842.9 & 844.9 & 847.7 & 845.8 & 850.5 \\
\hline 11.33 & 840.6 & 841.5 & 842.7 & 844.2 & 846.1 & 848.8 & 847.0 & 851.6 \\
\hline 11.48 DS & 841.6 & 842.4 & 843.6 & 845.1 & 847.0 & 849.6 & 847.8 & 852.3 \\
\hline 11.48 US & 842.2 & 843.6 & 845.4 & 846.1 & 847.4 & 849.7 & 848.0 & 852.5 \\
\hline 11.56 & 842.4 & 843.8 & 845.6 & 846.4 & 847.7 & 850.0 & 848.4 & 852.7 \\
\hline 11.74 & 843.3 & 844.5 & 846.2 & 847.3 & 848.9 & 851.3 & 849.6 & 854.0 \\
\hline 11.85 & 843.7 & 844.8 & 846.5 & 847.7 & 749.3 & 851.7 & 850.0 & 854.4 \\
\hline 12.09 & 845.1 & 846.0 & 847.4 & 848.6 & 850.4 & 852.7 & 851.1 & 855.4 \\
\hline 12.14 DS & 845.4 & 846.4 & 847.7 & 849.1 & 850.8 & 853.2 & 851.4 & 855.7 \\
\hline 12.14 US & 845.9 & 847.1 & 849.6 & 850.8 & 852.2 & 853.8 & 852.8 & 855.8 \\
\hline 12.18 & 846.3 & 847.5 & 849.9 & 851.4 & 853.1 & 855.4 & 853.8 & 857.5 \\
\hline 12.34 & 847.6 & 848.9 & 851.0 & 852.9 & 855.0 & 857.6 & 855.8 & 860.5 \\
\hline 12.38 & 847.9 & 849.1 & 851.2 & 853.1 & 855.1 & 857.7 & 856.0 & 860.7 \\
\hline 12.47 DS & 848.9 & 850.4 & 851.9 & 853.7 & 855.6 & 858.3 & 856.5 & 861.0 \\
\hline 12.47 US & 849.0 & 851.6 & 852.7 & 853.9 & 855.7 & 858.3 & 856.6 & 861.1 \\
\hline 12.55 & 849.6 & 851.9 & 853.0 & 854.2 & 855.8 & 858.5 & 856.6 & 861.2 \\
\hline $12.60 \mathrm{DS}$ & 849.8 & 852.1 & 853.4 & 855.1 & 857.0 & 859.7 & 858.0 & 862.0 \\
\hline 12.60 US & 850.4 & 852.8 & 854.4 & 855.8 & 857.6 & 859.9 & 858.3 & 862.0 \\
\hline 12.68 & 851.0 & 853.1 & 854.7 & 856.4 & 858.2 & 860.8 & 859.0 & 863.1 \\
\hline $12.89 \mathrm{DS}$ & 853.1 & 854.4 & 855.7 & 857.5 & 859.3 & 861.7 & 860.0 & 864.1 \\
\hline 12.89 US & 853.8 & 855.1 & 856.1 & 857.5 & 859.3 & 861.7 & 860.0 & 864.2 \\
\hline 13.02 & 855.7 & 856.6 & 857.5 & 858.8 & 860.4 & 862.6 & 861.0 & 864.8 \\
\hline $13.17 \mathrm{DS}$ & 857.4 & 858.0 & 858.9 & 860.2 & 861.8 & 863.9 & 862.5 & 866.0 \\
\hline 13.17 US & 858.5 & 859.0 & 859.5 & 860.4 & 861.8 & 863.9 & 862.5 & 866.1 \\
\hline 13.27 DS & 859.0 & 859.7 & 860.5 & 861.9 & 863.3 & 865.0 & 863.8 & 866.8 \\
\hline 13.27 US & 859.3 & 863.0 & 866.3 & 866.9 & 867.5 & 868.3 & 867.8 & 869.1 \\
\hline 13.32 & 859.7 & 863.1 & 866.3 & 866.9 & 867.6 & 868.5 & 867.9 & 869.3 \\
\hline 13.49 & 862.8 & 864.0 & 866.6 & 867.6 & 868.8 & 870.4 & 869.3 & 872.1 \\
\hline 13.55 & 863.8 & 864.6 & 866.8 & 867.8 & 869.1 & 870.9 & 869.7 & 872.7 \\
\hline 13.65 & 866.1 & 866.5 & 867.6 & 868.8 & 870.1 & 872.0 & 870.7 & 874.0 \\
\hline 13.74 & 868.8 & 869.1 & 869.6 & 870.6 & 871.8 & 873.5 & 872.3 & 875.3 \\
\hline 13.85 & 873.8 & 874.3 & 875.2 & 876.2 & 877.2 & 878.2 & 877.5 & 879.2 \\
\hline
\end{tabular}


EAST FORK POPLAR CREEK

Elevations for Specified Events (Continued)

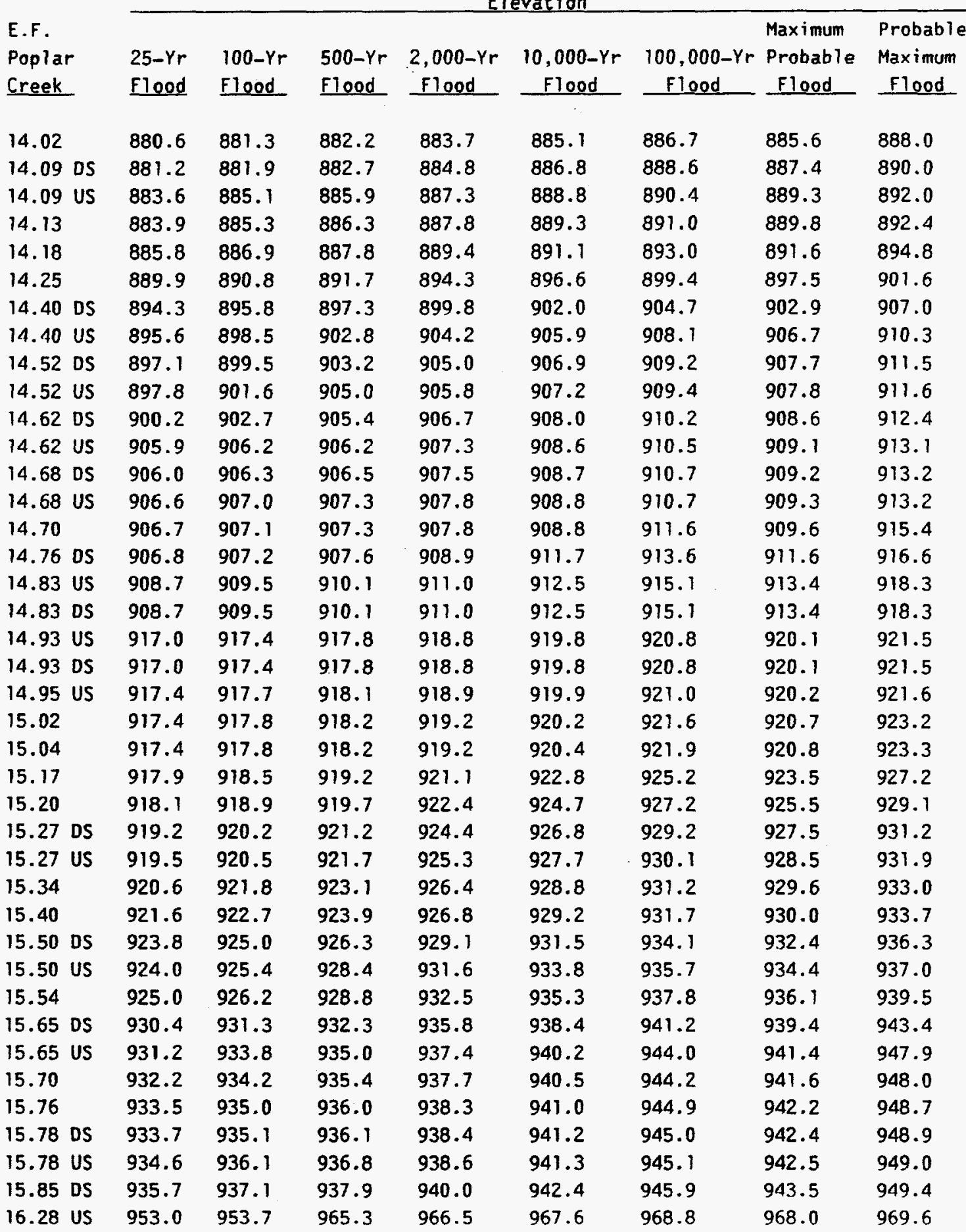


POPLAR CREEK

Elevations for Specified Events

\begin{tabular}{|c|c|c|c|c|c|c|c|c|}
\hline \multirow{2}{*}{ Poplar } & \multicolumn{8}{|c|}{ Elevation } \\
\hline & & & & & & & Maximum & Probable \\
\hline Creek & $25-Y r$ & $100-Y r$ & $500-Y r$ & $2,000-Y_{r}$ & $10,000-Y_{r}$ & $100,000-Y r$ & Probable & Maximum \\
\hline Mile & Flood & Flood & Flood & Flood & Flood & Flood & Flood & Flood \\
\hline 0.00 & $746.4^{\star}$ & $748.9^{\star}$ & $751.5^{\star}$ & $753.9^{*}$ & $757 \cdot 3^{\star \star}$ & $766.6^{\star x}$ & $759.4^{n \pi}$ & $777.3^{\star \star}$ \\
\hline 0.20 & $746.4^{\star}$ & $748.9^{\star}$ & $751.5^{\star}$ & $753.9^{\star}$ & $757.3^{\star \star}$ & $766.6^{\star \star \star}$ & $759.4^{\star \star}$ & $777.3^{\star \star}$ \\
\hline 0.60 & $746.4^{\pi}$ & $748.9^{*}$ & $751.5^{\star}$ & $753.9 *$ & $757.3^{* *}$ & $766.6^{* \star}$ & $759.4^{\star *}$ & $777.3^{\times x}$ \\
\hline 1.10 & $746.4^{\star}$ & $748.9^{\star}$ & $751.5^{\star}$ & $753.9^{\star}$ & $757.3^{* \star}$ & $766.6^{\star \star \star}$ & $759.4^{\star \star}$ & $777.3^{\star \star}$ \\
\hline 1.60 & $746.4^{*}$ & $748.9^{*}$ & $751.5^{*}$ & $753.9^{*}$ & $757.3^{* \star}$ & $766.6^{* \star}$ & $759.4^{\star \star}$ & $777.3^{* \star}$ \\
\hline 1.99 & $746.4^{*}$ & $748.9^{\star}$ & $751.5^{\star}$ & $753.9^{\star}$ & $757 \cdot 3^{\star \star}$ & $766.6^{\star \star}$ & $759.4^{\star \star *}$ & $777.3^{* \star}$ \\
\hline 2.27 & $746.4^{\star}$ & $748.9^{\star}$ & $751.5^{\star}$ & $753.9^{\star}$ & $757.3^{\star \star}$ & $766.6^{\star *}$ & $759.4^{\star \star \star}$ & $777.3^{\star x}$ \\
\hline $2.28 \mathrm{DS}$ & $746.4^{*}$ & $748.9^{*}$ & $751.5^{\star}$ & $753.9 *$ & $757.3^{* x}$ & $766.6 * *$ & $759.4^{* *}$ & $777.3^{\star \star}$ \\
\hline 2.28 US & $746.4^{\star}$ & $748.9^{\star}$ & $751.5^{\star}$ & $753.9^{\star}$ & $757.3^{\star \star}$ & $766.6^{\star \star}$ & $759,4^{\star *}$ & $777.3^{\star *}$ \\
\hline $2.50 \mathrm{DS}$ & $746.4^{*}$ & $748.9^{*}$ & $751.5^{\star}$ & $753.9^{\star}$ & $757.3^{\star \star}$ & $766.6^{\star \star}$ & $759.4^{* \star}$ & $777.3^{* \pi}$ \\
\hline 2.50 US & $746.4^{\star}$ & $748.9^{\star}$ & $751.5^{\star}$ & $753.9^{\star}$ & $757.3^{\star \star}$ & $766.6^{\star \star *}$ & $759.4^{\star \star \star}$ & $777.3^{\star \star}$ \\
\hline 2.80 & $746.4^{x}$ & $748.9 *$ & $751.5^{\star}$ & $753.9 *$ & $757 \cdot 3^{* *}$ & $766.6^{\star \star \star}$ & $759.4^{\star \star x}$ & $777.3^{\star \star}$ \\
\hline 2.84 & $746.4^{*}$ & 748.9* & $751.5^{\star}$ & $753.9 *$ & $757 \cdot 3^{* \star}$ & $766.6^{* \star}$ & $759.4^{* *}$ & $777.3^{* \star}$ \\
\hline 2.90 & 746.5 & $748.9^{\star}$ & 751.6 & $753.9^{\star}$ & $757 \cdot 3^{\star \star}$ & $766.6^{* *}$ & $759.4^{\star *}$ & $777.3^{\star \star}$ \\
\hline 2.96 & 746.6 & $748.9^{\star}$ & 751.7 & 754.0 & 757.4 & $766.6^{\star \star x}$ & $759.4^{\star \star}$ & $777.3^{\star \star}$ \\
\hline 3.17 DS & 746.9 & 749.3 & 752.1 & 754.5 & 757.5 & $766.6^{\text {** }}$ & $759.4^{* *}$ & $777.3^{\star \star x}$ \\
\hline 3.17 US & 747.2 & 749.6 & 752.5 & 755.0 & 758.0 & $766.6^{\star * *}$ & $759.4^{\star \star \star}$ & $777.3^{\star \star}$ \\
\hline 3.45 & 748.2 & 750.8 & 753.9 & 756.0 & 758.6 & $766.6^{\star \star *}$ & $759.4^{\star \star *}$ & $777.3^{* *}$ \\
\hline 3.60 & & 751.5 & & & 758.9 & $766.6^{* *}$ & 760.1 & $777.3^{* *}$ \\
\hline 4.40 & 750.9 & 754.0 & 757.6 & 759.6 & 762.1 & 768.2 & 763.4 & $777.3^{\star \star}$ \\
\hline $4.67 \mathrm{DS}$ & 751.6 & 754.9 & 758.6 & 760.7 & 763.4 & 769.3 & 764.8 & $777.3^{\star \star}$ \\
\hline 4.67 US & 751.8 & 755.2 & 758.9 & 761.1 & 763.8 & 769.8 & 765.3 & $777.3^{\text {** }}$ \\
\hline 4.73 OS & 752.0 & 755.4 & 759.1 & 761.3 & 764.0 & 769.9 & 765.5 & $777.3^{* *}$ \\
\hline 4.73 US & 752.2 & 755.7 & 759.6 & 761.8 & 764.5 & 770.3 & 766.0 & $777.3^{\star *}$ \\
\hline 4.97 & 753.0 & 756.5 & 760.5 & 762.7 & 765.4 & 770.7 & 766.8 & $777.3^{\star * *}$ \\
\hline 5.03 & 753.2 & 756.7 & 760.7 & 763.0 & 765.7 & 771.0 & 767.2 & $777.3^{\star \star}$ \\
\hline 5.29 & 754.2 & 757.8 & 761.8 & 764.1 & 767.0 & 772.2 & 768.3 & 778.7 \\
\hline 5.63 & 755.0 & 758.6 & 762.5 & 764.8 & 767.6 & 772.7 & 768.9 & 779.1 \\
\hline
\end{tabular}

* Controlling elevations are from the Clinch River

** Controlling elevations are from the Tennessee River. Tennessee River elevations reflect the recently completed dam safety modification at Fort LoudounTellico and the proposed dam safety modification at Watts Bar Dam which calis for raising the embankment 10 feet; these modifications enable these projects to safely pass the PMF. 
BEAR CREEK

Elevations for Specified Events

Elevation

\begin{tabular}{|c|c|c|c|c|c|c|c|c|}
\hline Bear & & & & & & & Maximum & Probable \\
\hline Creek & $25-Y r$ & $100-Y r$ & $500-Y r$ & $2,000-Y r$ & $10,000-Y r$ & $100,000-Y r$ & Probable & Maximum \\
\hline Mile & Flood & Flood & Flood & Flood & Flood & Flood & Flood & Flood \\
\hline 0.20 & $756.1 *$ & $758.3^{\star}$ & $762.2^{\star \star \star}$ & $764.5^{\star \star}$ & $767.3^{\star x}$ & $772.5^{\star \star}$ & $768.5^{\star x}$ & $778.9 * *$ \\
\hline 0.39 DS & $756.1^{*}$ & $758.3^{\star}$ & $762.2^{\star \star x}$ & $764.5^{* x}$ & $767 \cdot 3^{\star \star}$ & $772.5^{\star \star *}$ & $768.5^{\star \star}$ & $778.9 * *$ \\
\hline 0.39 US & $756.1^{*}$ & $758.3^{\star}$ & $762.2^{\star *}$ & $764.5^{* \star}$ & $767.3^{* *}$ & $772.5^{\star *}$ & $768.5^{* *}$ & $778.9 * *$ \\
\hline 0.55 DS & $756.1^{*}$ & $758.3^{*}$ & $762.2^{\star *}$ & $764.5^{\star \star}$ & $767.3^{\star *}$ & $772.5^{\star \star}$ & $768.5^{\star \star}$ & $778.9^{* x}$ \\
\hline 0.55 US & $756.1^{\star}$ & $758.3^{*}$ & $762.2^{\star \star *}$ & $764.5^{\star \star}$ & $767.3^{\star *}$ & $772.5^{\star \star}$ & $768.5^{\star \star}$ & $778.9 * *$ \\
\hline 0.61 & $756.1^{*}$ & $758.3^{\star}$ & $7.62 .2^{\star \pi}$ & $764.5^{\pi \star}$ & $767.3^{\star *}$ & $772.5^{\star \star}$ & $768.5^{\star \star}$ & $778.9 * x$ \\
\hline 0.65 & 756.8 & $758.3^{\star}$ & $762.2^{\star *}$ & $764.5^{* *}$ & $767.3^{\star \star *}$ & $772.5^{\star \star}$ & $768.5^{* \star}$ & $778.9^{\star \star *}$ \\
\hline 0.70 & 757.8 & $758.3^{\star}$ & $762.2^{* *}$ & $764.5^{* \star}$ & $767.3^{\star \star x}$ & $772.5^{\star \star}$ & $768.5^{\star \star}$ & $778.9^{x *}$ \\
\hline 0.77 & 759.1 & 759.8 & $762.2^{\star *}$ & $764.5^{\star \star}$ & $767.3^{\star \star *}$ & $772.5^{\star \star}$ & $768.5^{\star \star}$ & $778.9 * *$ \\
\hline 0.78 & 759.3 & 759.9 & $762.2^{\star \star}$ & $764.5^{\star *}$ & $767.3^{\star \star}$ & $772.5^{\star \star}$ & $768.5^{\star \star}$ & $778.9 \star \star$ \\
\hline 0.91 & 760.8 & 761.5 & $762.2^{\times x}$ & $764.5^{* \pi}$ & $767.3^{\times x}$ & $772.5^{\star *}$ & 770.0 & $778.9^{* *}$ \\
\hline 0.92 DS & 760.9 & 761.6 & 762.3 & $764.5^{\star *}$ & $767.3^{\star *}$ & $772.5^{\star \star}$ & 770.1 & $778.9^{\text {* }}$ \\
\hline 0.92 US & 761.1 & 762.0 & 763.0 & $764.5^{m \pi}$ & $767.3^{\star \star}$ & $772.5^{\star \star}$ & 770.3 & $778.9^{* *}$ \\
\hline $1.08 \mathrm{DS}$ & 762.5 & 763.2 & 764.1 & 766.3 & 769.8 & 774.2 & 771.4 & $778.9^{* \star}$ \\
\hline 1.08 US & 762.8 & 763.6 & 764.5 & 767.3 & 770.2 & 775.0 & 772.9 & $778.9 \times x$ \\
\hline 1.27 & 765.3 & 765.8 & 766.4 & 768.6 & 772.2 & 777.0 & 774.4 & $778.9^{* \star}$ \\
\hline $1.28 \mathrm{DS}$ & 765.4 & 765.9 & 766.5 & 768.8 & 772.4 & 777.3 & 774.4 & 779.0 \\
\hline 1.28 US & 765.9 & 766.5 & 767.1 & 769.5 & 773.2 & 777.4 & 774.5 & 779.1 \\
\hline $1.50 \mathrm{DS}$ & 769.5 & 769.8 & 770.2 & 771.5 & 774.4 & 778.2 & 775.9 & 780.2 \\
\hline 1.50 US & 769.8 & 770.2 & 770.6 & 774.2 & 778.4 & 780.3 & 780.0 & 780.5 \\
\hline 2.37 & 788.8 & 789.2 & 789.6 & 790.2 & 792.6 & 795.1 & 793.9 & 797.0 \\
\hline $2.78 \mathrm{DS}$ & 801.1 & 801.6 & 802.0 & 803.5 & 805.9 & 808.0 & 806.4 & 809.3 \\
\hline 2.78 US & 801.3 & 801.8 & 802.2 & 805.0 & 808.7 & 811.0 & 809.9 & 811.9 \\
\hline $2.91 \mathrm{DS}$ & 804.5 & 804.8 & 805.1 & 806.1 & 809.2 & 811.4 & 810.5 & 812.8 \\
\hline 2.91 US & 804.6 & 805.0 & 805.3 & 808.8 & 813.4 & 816.8 & 815.2 & 817.2 \\
\hline 3.17 & 807.1 & 807.4 & 807.9 & 810.8 & 814.3 & 817.0 & 815.7 & 818.3 \\
\hline 3.52 & 818.3 & 818.7 & 819.0 & 821.2 & 823.3 & 825.7 & 824.0 & 827.2 \\
\hline 3.92 DS & 831.5 & 832.2 & 832.6 & 833.7 & 835.8 & 839.1 & 837.0 & 840.0 \\
\hline 3.92 US & 831.9 & 832.6 & 833.0 & 834.5 & 837.2 & 841.3 & 838.5 & 841.3 \\
\hline 4.36 DS & 839.5 & 839.9 & 840.2 & 842.1 & 844.7 & 847.0 & 845.4 & 848.7 \\
\hline 4.36 US & 839.7 & 841.5 & 842.5 & 844.0 & 846.9 & 849.0 & 847.9 & 849.7 \\
\hline 4.74 & 845.4 & 845.7 & 846.1 & 847.8 & 850.4 & 852.7 & 851.3 & 854.3 \\
\hline $5.04 \mathrm{DS}$ & 854.5 & 854.9 & 855.1 & 856.6 & 858.7 & 860.5 & 859.2 & 862.2 \\
\hline 5.04 US & 856.0 & 857.4 & 858.4 & 859.2 & 860.7 & 862.2 & 861.4 & 862.9 \\
\hline 5.27 & 858.0 & 858.5 & 859.1 & 860.4 & 861.9 & 864.1 & 863.0 & 865.4 \\
\hline 5.71 & 873.6 & 874.1 & 874.4 & 875.2 & 876.9 & 879.0 & 878.0 & 880.5 \\
\hline 6.03 DS & 886.6 & 886.9 & 887.2 & 888.0 & 889.7 & 892.2 & 890.9 & 893.3 \\
\hline 6.03 US & 890.7 & 891.0 & 891.0 & 891.9 & 892.5 & 893.8 & 892.9 & 894.0 \\
\hline $6.38 \mathrm{DS}$ & 900.5 & 900.8 & 901.0 & 901.8 & 902.9 & 904.2 & 903.7 & 905.3 \\
\hline 6.38 US & 902.1 & 902.3 & 902.4 & 903.1 & 904.1 & 905.5 & 904.4 & 906.2 \\
\hline
\end{tabular}

* Controlling elevations are from East Fork Poplar Creek

* Controlling elevations are from Poplar Creek 


\section{BEAR CREEK}

Elevations for Specified Events (Continued)

\begin{tabular}{|c|c|c|c|c|c|c|c|c|}
\hline \multirow[b]{2}{*}{ Bear } & \multicolumn{8}{|c|}{ Elevation } \\
\hline & & & & & & & Maximum & Probable \\
\hline Creek & $25-Y r$ & $100-Y r$ & $500-Y r$ & $2,000-Y r$ & $10,000-Y r$ & $100,000-Y_{r}$ & Probable & Maximum \\
\hline Mile & Flood & Flood & Flood & Flood & Flood & Flood & Flood & Flood \\
\hline $6.59 \mathrm{DS}$ & 908.0 & 908.3 & 908.5 & 909.1 & 910.2 & 911.0 & 910.8 & 911.9 \\
\hline 6.59 US & 909.8 & 910.2 & 910.4 & 911.0 & 912.5 & 913.9 & 913.1 & 914.4 \\
\hline 6.73 & 911.3 & 911.5 & 911.7 & 912.5 & 914.0 & 915.7 & 914.9 & 916.8 \\
\hline 7.20 & 927.7 & 927.9 & 928.0 & 928.7 & 929.5 & 930.5 & 929.8 & 931.1 \\
\hline $7.35 \mathrm{dS}$ & 938.2 & 938.4 & 938.6 & 939.1 & 940.6 & 941.9 & 941.2 & 942.8 \\
\hline 7.35 US & 939.1 & 939.3 & 939.5 & 942.5 & 946.0 & 947.9 & 947.2 & 948.4 \\
\hline 7.52 & 944.4 & 944.7 & 945.0 & 946.0 & 948.0 & 950.2 & 949.1 & 951.4 \\
\hline $7.70 \mathrm{DS}$ & 952.2 & 952.5 & 952.8 & 954.0 & 955.5 & 956.7 & 956.2 & 957.3 \\
\hline 7.70 US & 954.7 & 954.9 & 956.3 & 956.5 & 957.1 & 958.0 & 957.5 & 958.8 \\
\hline $7.78 \mathrm{DS}$ & 956.7 & 956.9 & 957.3 & 957.9 & 959.4 & 961.0 & 960.2 & 961.9 \\
\hline 7.78 US & 957.0 & 957.3 & 957.6 & 959.7 & 962.5 & 964.0 & 963.5 & 964.2 \\
\hline 7.98 & 967.1 & 967.3 & 967.6 & 968.4 & 970.0 & 971.2 & 970.6 & 972.1 \\
\hline 8.14 DS & 979.6 & 979.7 & 979.9 & 981.0 & 982.7 & 984.0 & 983.2 & 984.8 \\
\hline 8.14 US & 980.8 & 981.0 & 981.2 & 985.0 & 992.4 & 994.5 & 994.1 & 994.8 \\
\hline
\end{tabular}




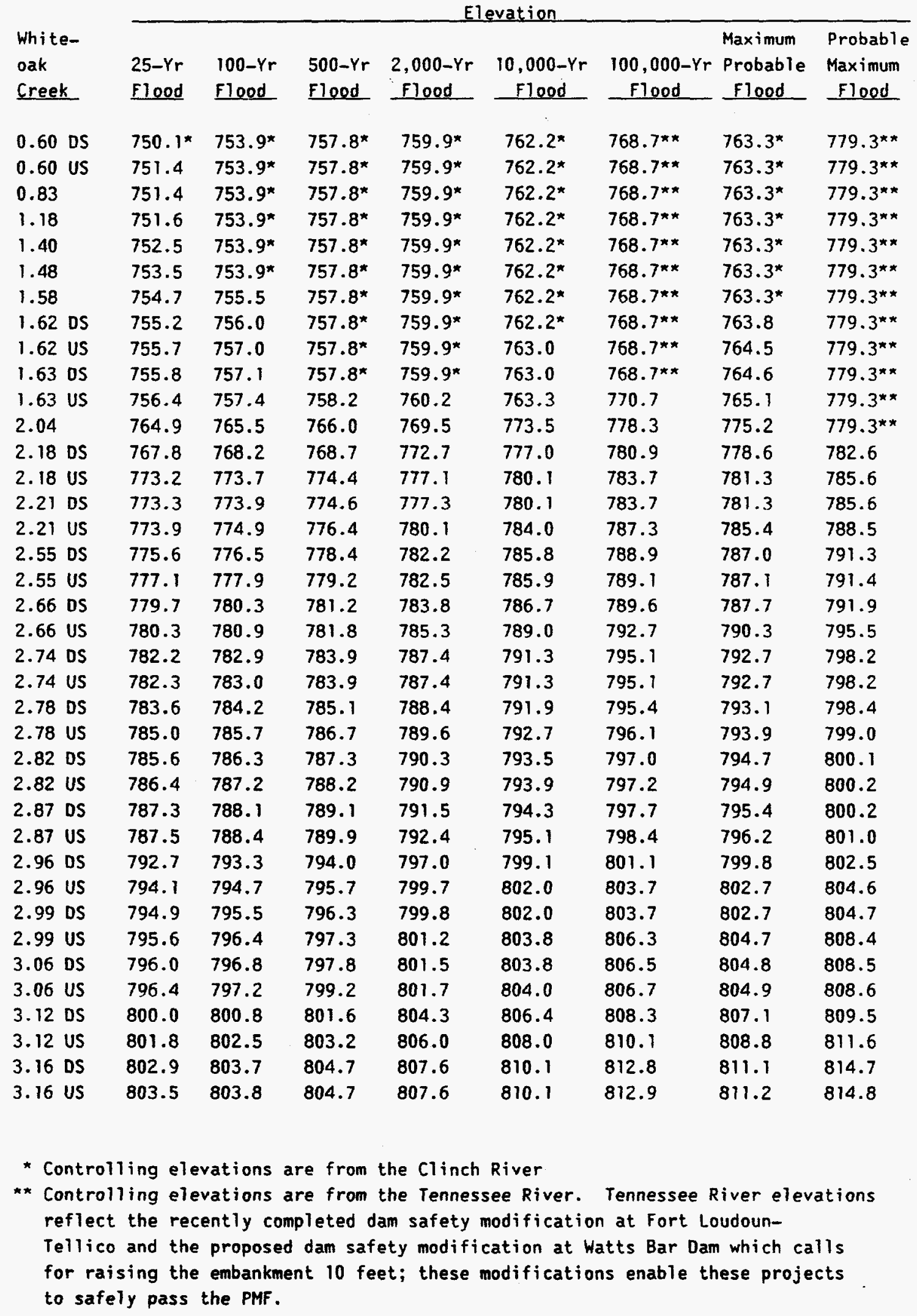


WHITEOAK CREEK

Elevations for Specified Events (Continued)

\begin{tabular}{|c|c|c|c|c|c|c|c|c|}
\hline \multirow[b]{2}{*}{ White- } & \multicolumn{8}{|c|}{ Elevation } \\
\hline & & & & & & & Maximum & Probable \\
\hline & $25-Y r$ & $100-Y r$ & $500-Y r$ & $2,000-Y r$ & $10,000-Y_{r}$ & $100,000-Y r$ & Probable & Maximum \\
\hline Creek & Flood & Flood & Flood & Flood & Flood & Flood & Flood & Flood \\
\hline 3.24 DS & 805.2 & 805.7 & 805.9 & 808.4 & 811.0 & 813.5 & 811.9 & 815.4 \\
\hline 3.24 US & 809.3 & 810.1 & 810.4 & 812.4 & 814.2 & 815.9 & 814.7 & 817.0 \\
\hline 3.47 DS & 819.1 & 820.0 & 820.6 & 822.7 & 824.4 & 826.2 & 825.0 & 827.3 \\
\hline 3.47 US & 820.5 & 825.8 & 826.3 & 827.9 & 829.3 & 830.5 & 829.8 & 831.4 \\
\hline 3.55 DS & 823.5 & 825.9 & 826.4 & 828.0 & 829.7 & 831.2 & 830.1 & 832.1 \\
\hline 3.55 US & 826.9 & 827.1 & 827.3 & 828.8 & 830.0 & 831.5 & 830.3 & 832.2 \\
\hline 3.78 & 838.3 & 839.3 & 839.8 & 841.8 & 843.6 & 845.4 & 844.1 & 846.4 \\
\hline 4.00 & 852.7 & 852.9 & 853.7 & 855.5 & 857.3 & 859.4 & 858.0 & 861.0 \\
\hline
\end{tabular}


MELTON BRANCH

Elevations for Specified Events

\begin{tabular}{|c|c|c|c|c|c|c|c|c|}
\hline \multirow{2}{*}{ Melton } & \multicolumn{8}{|c|}{ Elevation } \\
\hline & & & & & & & Maximum & Probable \\
\hline Branch & $25-Y r$ & $100-Y r$ & $500-Y r$ & $2,000-Y r$ & $10,000-Y_{r}$ & $100,000-\gamma_{r}$ & Probable & Maximum \\
\hline Mile & Flood & Flood & flood & Flood & Flood & Flood & Flood & Flood \\
\hline 0.00 & $754.3^{\star}$ & $755.2^{\star}$ & $757.8^{* \star}$ & $759.9^{\star}$ & $762.2^{\star}$ & $768.7 * * *$ & $763.3^{\star \star}$ & $779 \cdot 3^{* * *}$ \\
\hline $0.11 \mathrm{DS}$ & 754.9 & 755.3 & $757.8 * \star$ & $759.9^{\star}$ & $762.2^{*}$ & $768.7^{\star \star \star *}$ & $763.3^{\star *}$ & $779.3^{* x *}$ \\
\hline 0.11 US & 758.3 & 759.3 & 759.9 & 760.5 & 762.5 & $768.7^{\star * *}$ & $763.3^{\star \star}$ & $779.3^{\star \star \star}$ \\
\hline 0.15 & 758.8 & 759.7 & 760.3 & 761.0 & 763.0 & 768.9 & $763.3^{\star \star}$ & $779 \cdot 3^{\text {x*x }}$ \\
\hline 0.25 & 759.9 & 760.7 & 761.3 & 762.2 & 764.3 & 769.5 & 765.4 & $779.3^{* * *}$ \\
\hline 0.39 & 764.0 & 764.6 & 765.2 & 766.4 & 769.0 & 773.4 & 770.5 & $779.3^{* * *}$ \\
\hline 0.56 & 770.7 & 771.2 & 771.9 & 773.4 & 775.5 & 778.3 & 776.5 & $779.3^{* * *}$ \\
\hline $0.67 \mathrm{DS}$ & 775.1 & 775.4 & 776.3 & 777.9 & 779.7 & 781.4 & 780.3 & 782.9 \\
\hline 0.67 US & 777.8 & 778.1 & 778.4 & 779.9 & 781.6 & 783.2 & 782.2 & 784.4 \\
\hline 0.85 & 782.4 & 783.4 & 784.2 & 785.9 & 787.7 & 789.4 & 788.4 & 790.8 \\
\hline $1.12 \mathrm{DS}$ & 794.0 & 794.5 & 794.9 & 796.8 & 799.1 & 801.2 & 800.1 & 803.0 \\
\hline 1.12 US & 794.9 & 795.2 & 795.6 & 797.2 & 799.6 & 801.8 & 800.5 & 803.3 \\
\hline $1.48 \mathrm{DS}$ & 803.6 & 804.1 & 804.6 & 806.4 & 809.0 & 811.1 & 810.0 & 812.5 \\
\hline 1.48 US & 806.2 & 806.7 & 807.1 & 808.7 & 810.1 & 811.9 & 810.8 & 813.2 \\
\hline 1.58 & 810.2 & 810.6 & 810.9 & 813.3 & 815.0 & 816.9 & 815.8 & 818.2 \\
\hline 1.67 & 817.1 & 817.4 & 817.7 & 818.9 & 820.3 & 821.8 & 821.0 & 822.9 \\
\hline 1.93 & 838.7 & 838.9 & .839 .0 & 840.0 & 841.1 & 842.4 & 841.7 & 843.3 \\
\hline 2.05 & 867.3 & 867.5 & 867.6 & 869.3 & 871.4 & 872.8 & 872.1 & 873.8 \\
\hline
\end{tabular}

Melton

Branch

Trib. at

Mile 1.55

$\begin{array}{lllllllll}0.00 & 809.0 & 809.4 & 809.8 & 811.9 & 813.5 & 815.4 & 814.3 & 816.7 \\ 0.11 & 816.0 & 816.3 & 816.7 & 818.2 & 820.2 & 821.9 & 821.0 & 822.9 \\ 0.27 \text { OS } & 831.4 & 831.5 & 831.6 & 832.5 & 833.7 & 834.8 & 834.1 & 835.5 \\ 0.27 \text { US } & 832.0 & 832.1 & 832.4 & 835.3 & 839.0 & 841.0 & 840.5 & 841.7\end{array}$

\footnotetext{
* Controlling elevations are from Whiteoak Creek

** Controlling elevations are from the Clinch River

*** Controlling elevations are from the Tennessee River. Tennessee River elevations reflect the recently completed dam safety modification at Fort LoudounTellico and the proposed dam safety modification at Watts Bar Dam which calls for raising the embankment 10 feet; these modifications enable these projects to safely pass the PMF.
} 


\begin{tabular}{|c|c|c|c|c|c|c|c|c|}
\hline \multirow[b]{2}{*}{ Duration } & \multicolumn{6}{|c|}{ Rainfall } & \multirow{2}{*}{$\begin{array}{l}\text { TVA } \\
\text { MPP } \\
\end{array}$} & \multirow[b]{2}{*}{ PMP } \\
\hline & $25-Y_{r}$ & $100-\gamma_{r}$ & $500-Y_{r}$ & $2,000-Y_{r}$ & $10,000-Y_{r}$ & $100,000-Y_{r}$ & & \\
\hline 5-minute & 0.64 & 0.77 & 1.0 & 1.3 & 1.7 & 2.4 & $\begin{array}{l}1.9 \\
3.5\end{array}$ & \\
\hline 15-minute & 1.34 & 1.63 & 2.35 & 3.2 & 4.3 & 5.9 & 4.8 & 8 \\
\hline 30 -minute & 1.92 & 2.37 & 3.3 & 4.5 & 6.4 & 9.1 & 7.2 & \\
\hline
\end{tabular}

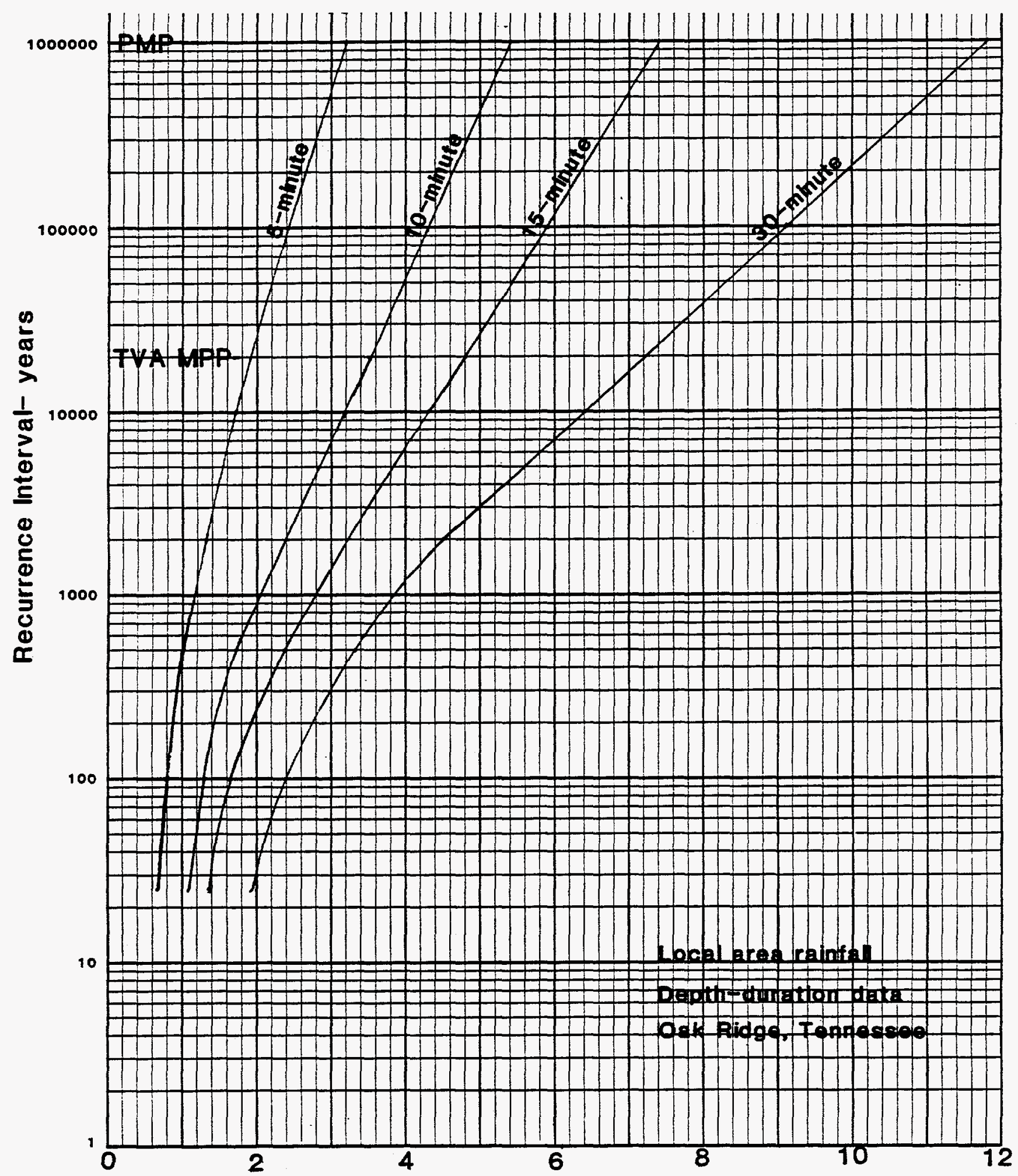




\begin{tabular}{|c|c|c|c|c|c|c|c|c|}
\hline \multirow[b]{2}{*}{ Duration } & \multicolumn{6}{|c|}{ Rainfall } & \multirow{2}{*}{$\begin{array}{r}\text { TVA } \\
\text { MPP } \\
\end{array}$} & \\
\hline & $25-Y_{r}$ & $100-Y_{r}$ & $500-Y_{r}$ & $2,000-Y_{r}$ & $10,000-Y_{r}$ & $100,000-Y_{r}$ & & \\
\hline $\begin{array}{l}\text { 1-hour } \\
\text { 2-hour }\end{array}$ & $\begin{array}{l}2.53 \\
3.0\end{array}$ & $\begin{array}{l}3.15 \\
3.75\end{array}$ & $\begin{array}{l}4.7 \\
5.7\end{array}$ & $\begin{array}{l}6.8 \\
8.5\end{array}$ & $\begin{array}{r}9.5 \\
12.4\end{array}$ & $\begin{array}{l}13.4 \\
18.3\end{array}$ & $\begin{array}{l}10.6 \\
14.1\end{array}$ & \\
\hline $\begin{array}{l}\text { 3-hour } \\
\text { 6-hour }\end{array}$ & $\begin{array}{l}3.4 \\
3.9\end{array}$ & $\begin{array}{l}4.0 \\
4.9\end{array}$ & $\begin{array}{l}6.9 \\
8.2\end{array}$ & $\begin{array}{l}10.2 \\
12.3\end{array}$ & $\begin{array}{l}14.7 \\
18.1\end{array}$ & $\begin{array}{l}21.2 \\
27.0\end{array}$ & $\begin{array}{l}16.6 \\
20.8\end{array}$ & \\
\hline
\end{tabular}

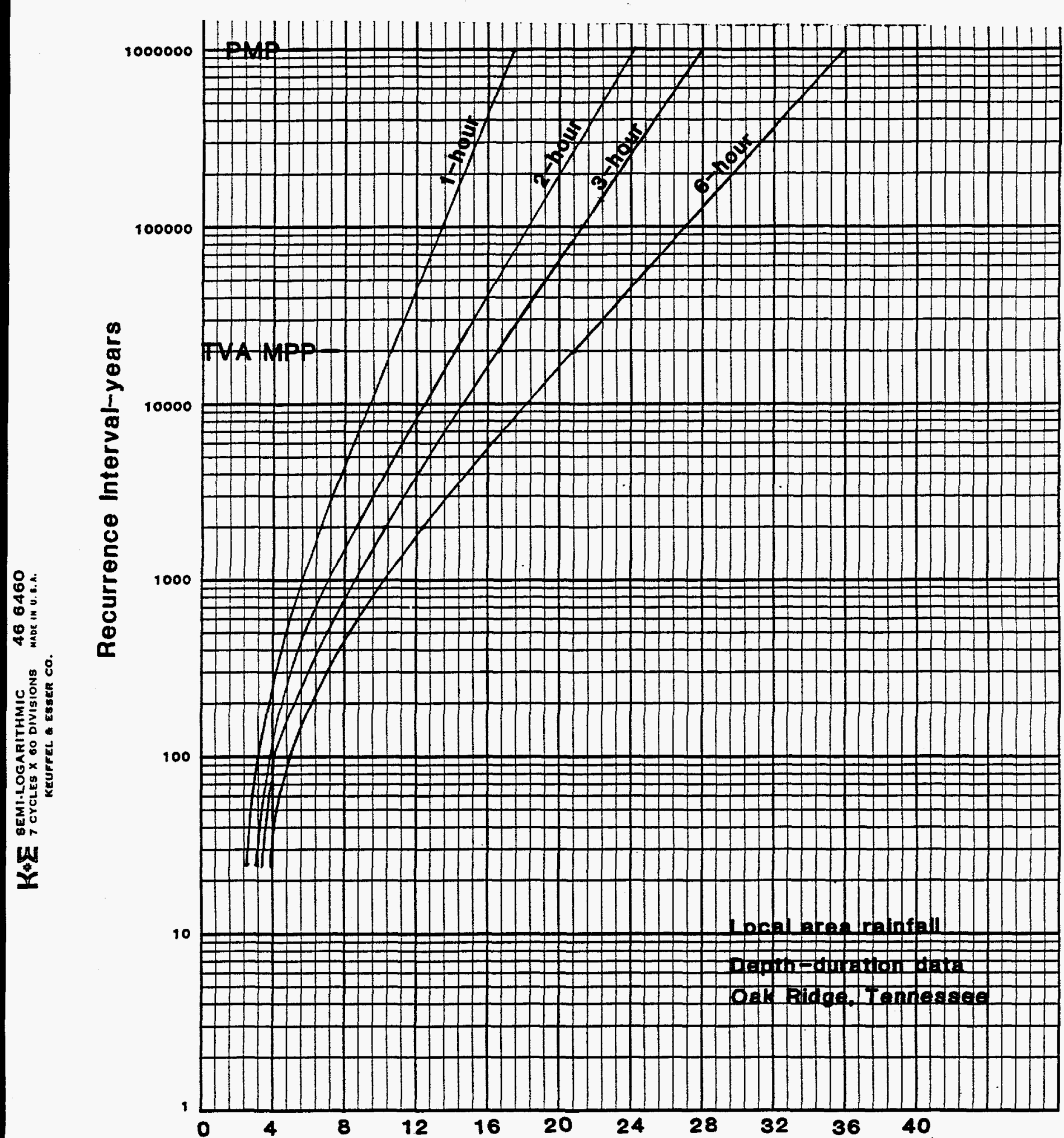


Department of Energy

Oak Ridge Operations

P.O. Box 2001

Oak Ridge, Tennessee $37831-8614$

November 5, 1991

Dr. Ralph Brooks, Manager

Division of Water Resources

Tennessee Valley Authority

Evans Building, Room 1W-141A

Knoxville, Tennessee 37902-1499

Dear Dr. Brooks:

\section{FLOOD ANALYSES IN SUPPORT OF FLOOD EMERGENCY PLANNING (INTERAGENCY AGREEMENT NO. DE-AI05-910R21979)}

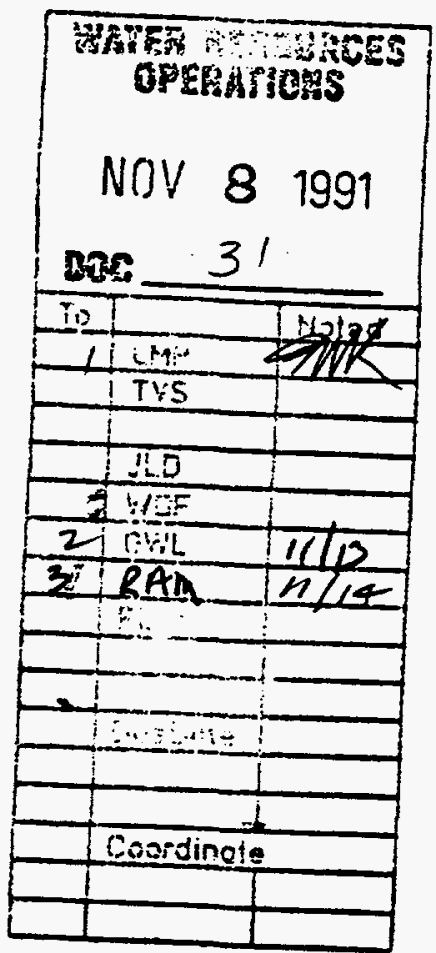

The Department of Energy (DOE) desires to modify the subject interagency agreement to extend the term of the agreement through December 31, 1991, with no additional funding or change in scope of work. This will permit the continuous technical support and assistance of the Tennessee Valley Authority (TVA) in connection with performing flood analyses in support of flood emergency planning.

Enclosed are two copies of the proposed amendment to the interagency agreement to accomplish this change. The amendment has been signed on behalf of DOE. If the amendment is acceptable to TVA, please sign the amendment on behalf of TVA and return one fully executed original to M. W. Henderson, ER-113.

Any questions regarding this proposed amendment should be directed to M. W. Henderson (FTS) 626-0714 or commercial number (615) 576-0714.

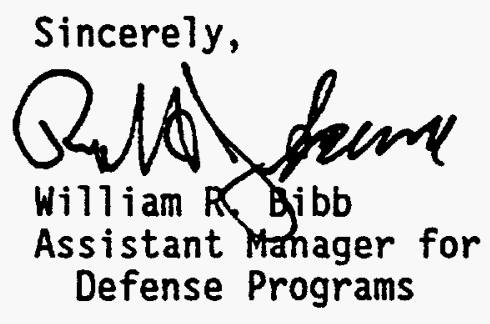

ER-113:Henderson

Enclosure:

Amendment M001

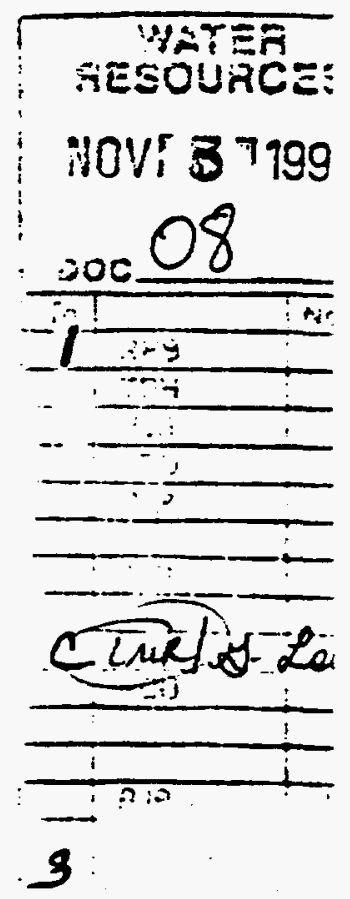


DOE Form 1270.2 (9-10)
U.S. DEPARTMENT OF ENERGY

INTERAGENCY AGREEMENT FACE PAGE

\begin{tabular}{|c|c|c|c|}
\hline $\begin{array}{l}\text { EUNDS-OUT INTERAGENCY AGRE } \\
\text { zursuant to authority of ECO } \\
\text { as amended ( } 31 \text { US.C. } 153 \\
\text { ther: }\end{array}$ & $\begin{array}{l}\text { TUA } \\
\text { ACt of } 1932 \text {, } \\
\text { PL } 95-91\end{array}$ & \multirow{2}{*}{$\begin{array}{l}\text { 1. IOENTIFICATION } \\
\text { a. DOE LA No.: } \\
\text { b. Other agency LA . Vo.: } \\
\text { c. Hlodibeation No.: } \\
\text { d. Task order : Yo.: } \\
\text { e. PR No. }\end{array}$} & \multirow[t]{2}{*}{$\begin{array}{l}\text { DE-AI05-910R21979 } \\
\text { TV-83730V } \\
\text { MO01 }\end{array}$} \\
\hline $\begin{array}{l}\text { TYPE OF ACTIONz } \\
\text { Now Awara I moanertion }\end{array}$ & Extension & & \\
\hline
\end{tabular}

3. PROJECT TITLENDESCRIPTIONI

Flood Analyses in Support of Flood Emergency Planning (No-cost extension)

IOENTIFICATION

a. DOE lA Vo.:

E-AI05-910R21979

c. Hodibeation No.: MOOI

d. Task order : Yo.

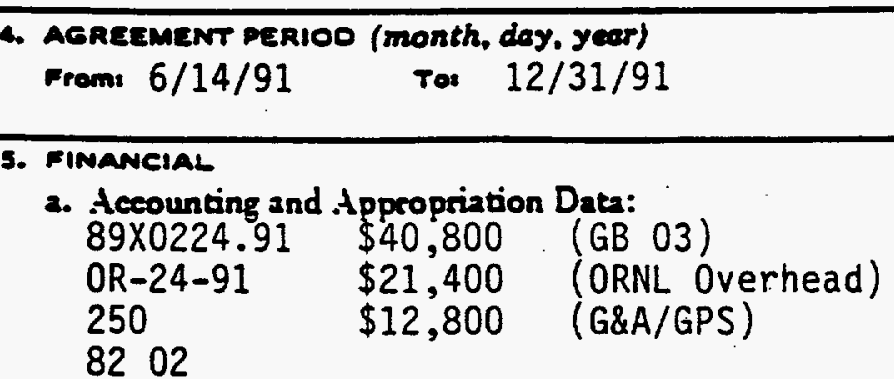

b. Funding sources

Basic Agreement

ooe This Action

Apenoy

Toeal Funaing

c. Hethod of Payment:

Anence

Q8 Retmoursement

Progress

d. Amount obligeted this action:

e. Invoices, if any, submit to:

Depertument of Enery.

P.0. Box 2001

Oak Ridge, TN 37831-8614

f. ATTN: Ficher 'W. Henderson, ER-113

SF-1080 and cite thereon

DE-AI05-910R21979 and Work Orders as stated in. Section IV Cost.
6. DOE PROGRAM OFFICER

Names Daniel Hoag

Y-12 Environmenta 1, Safety, and

Acaress:

Health Branch, DP- 813

U.S. Department of Energy

P.0. Box 2001 .

Rak Ridge, Tennessee 37831

(615) 576-0511 or (FTS) 626-0511

7. PERFORMINO AGENCY

a. Namas

Tennessee Valley Authority

Evans Bldg., Room 1W-141A

D. Acarens

Knoxville, TN 37902-1499

Attentton: Dr. Ralph Brooks, Manager Division of Water Resources

c. Program Director

Names

Adoreas: See 7.b.

Teteonone No

(615) $632-6770$

-. ISSUING AGENCY:

Department of Energy (DOE)

Field Office, Oak Ridge

P.0. Box 2001

Oak Ridge, Tennessee 37831-8600

\section{(aignature)}

(date)

10. DOE CONTRACTING OFFICER:

Yame (typewritten):

Title (spowricten):

Telephone:

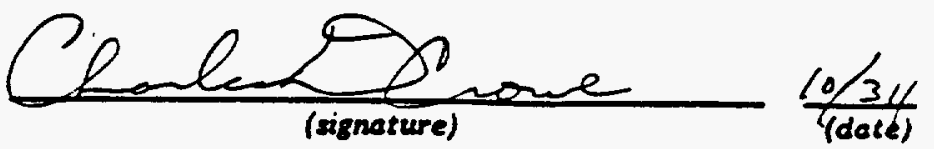

Name (typewritten): Charles D. Crowe Contracting Officer Environmental Acquisition $B_{1}$ Title (typewritten): Procurement \& Contracts Div. 


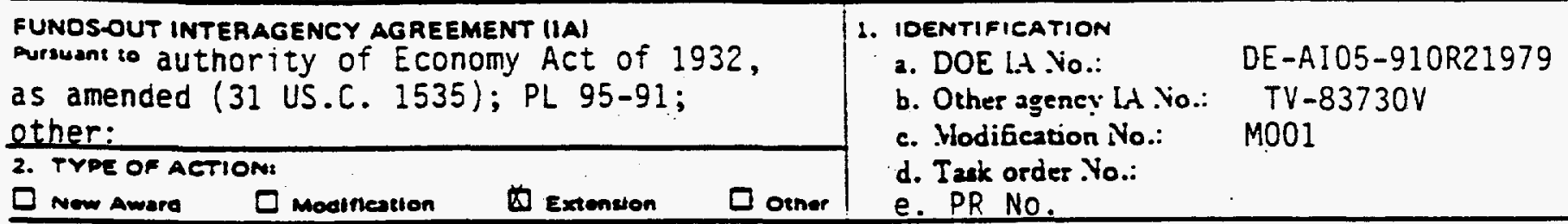

3. PROUECT TITLENOESCRIPTIONi

Flood Analyses in Support of Flood Emergency Planning (No-cost extension)

4. AGREEMENT PERIOO (month, dOY, yCar)

From: $6 / 14 / 91$

To: $12 / 31 / 91$

5. Financial

a. Aceounting and Appropriation Deta:

$\begin{array}{lll}89 \times 0224.91 & \$ 40,800 & (\text { GB O3) } \\ 0 R-24-91 & \$ 21,400 & \text { (ORNL Overhead) } \\ 250 & \$ 12,800 & \text { (G\&A/GPS) } \\ 8202 & & \end{array}$

b. Funding sources

Basic Agreement

Doe This Action

Ageney

Totas Funding

c. Hethod of Payment:

Aovence Do Rolmoursomem

Propress

d. Amount obligated this action:

$+0$

e. Invoices, if any, submit to:

Dopartment of Enory

P. D. Box 2001

Oak Ridge, TN 37831-8614

f. ATTN: An. WW Henderson, ER-113

SF -1080 and cite thereon

DE-AIO5-910R21979 and Work Orders

as stated in. Section IV Cost.

9. performine ageney aceeptance:

(signoture)

(date)

Yame (typewritten):

Title (typewitter):

Telepinone:
6. Doe procram officer

Momes Daniel Hoag

Y-12 Environmental, Safety, and

Acaress: Health Branch, DP-813

U.S. Department of Energy

P. 0. Box 2001

Oak Ridge, Tennessee 37831

(615) 576-0511 or (FTS) 626-0511

7. PERFORMING AGENCY

จ. Name:

Tennessee Valley Authority Evans Bldg., Room 1W-141A

o. Adaress: Knoxville, TN 37902-1499

Attentions Dr. Ralph Brooks, Manager

Division of Water Resources

c. Program Director

Name:

Acores: $\quad$ See 7.6

Tetepnone No

(615) $632-6770$

d. Issuing ageney:

Department of Energy (DOE)

Field Office, Oak Ridge

P.0. Box 2001

Oak Ridge, Tennessee 37831-8600

10. DOE CONTRACTING OFFICER:

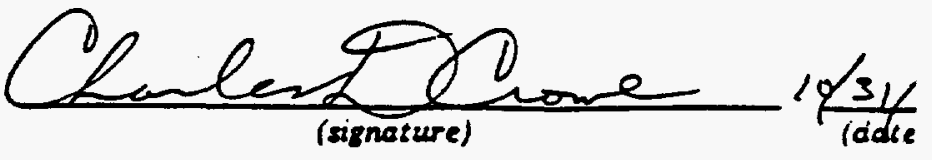

Vame (typewritten): Charles D. Crowe Contracting Officer

Titie (rypewritter): Environmental Acquisitions I Procurement \& Contracts Div 


\section{COVER SHEET TO BE USED FOR ALL WATER RESOURCES FORHS (EXCEPT TRAINING) AFTER RALPH H. BROOKS' AND \\ DEPARTMENT MANAGER'S SIGNATURE}

ORIGINAL FORM AND ATTACHMENTS MAILED TO:

Nancy J. Breeding, OCH IF 72E-K

Contract Services, OCH 2 G 95B-K

Central Payroll section, ET DI 30C-K

Aquatic Biology

Linda E. Hendon

Glenna H. Holder

Teresa V. Stooksbury

I copy of form and a copy of this sheet for Water Resources' reading files

Mailed on: $5,23,91$

Completed by:
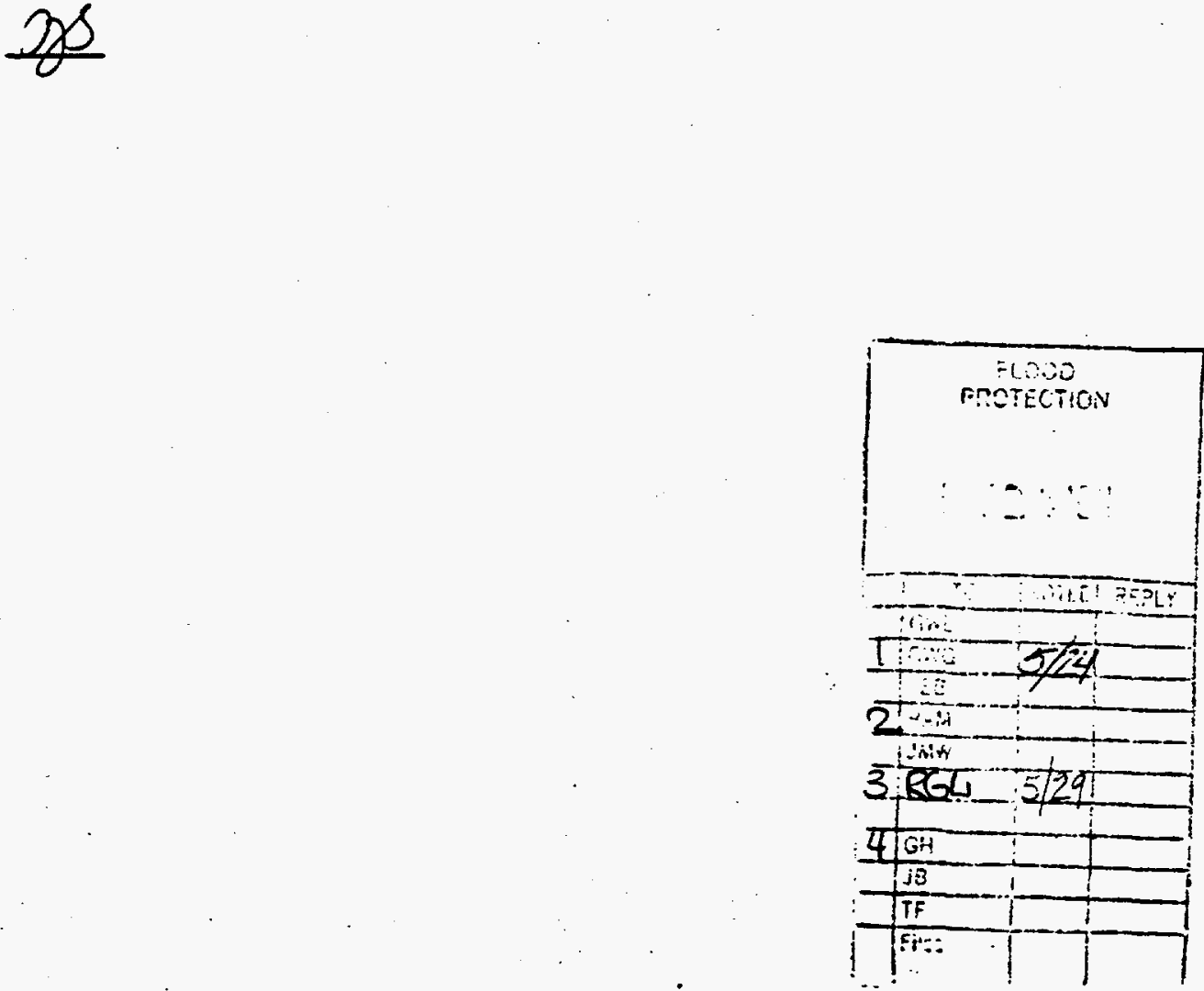
Department of Energy

Oak Ridge Operations

P.O. Box 2001

Oak Ridge, Tennessee 37831- 8614

May 7, 1991

Dr. Ralph Brooks, Manager

Division of Water Resources

Tennessee Valley Authority

Evans Building, Room 1W-141A

Knoxville, Tennessee 37902-1499

Dear Dr. Brooks:

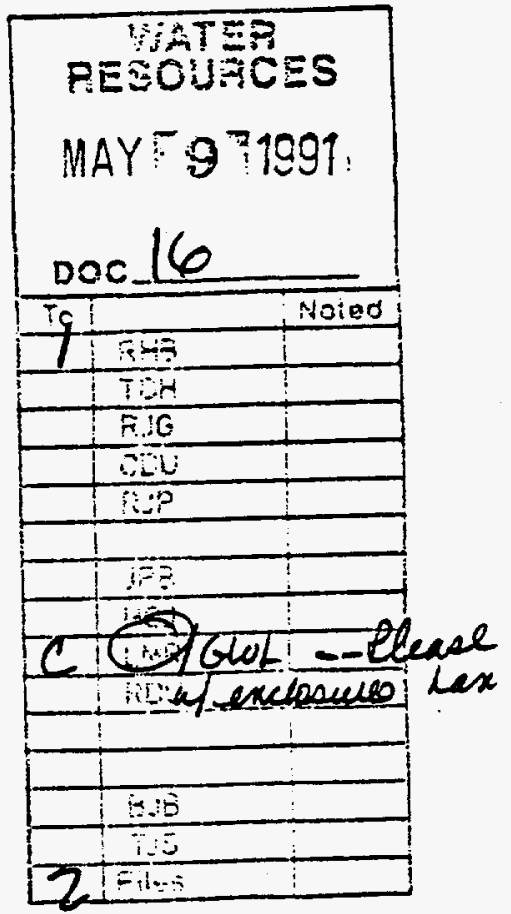

FLOOD ANALYSES IN SUPPORT OF FLOOD EMERGENCY PLANNING (INTERAGENCY AGREEMENT NO. DE-AI05-910R21979)

The Department of Energy (DOE) has a need for the technical support and assistance of the Tennessee Valley Authority (TVA) in connection with performing flood analyses in support of flood emergency planning.

Section IV of this agreement provides for a budget of $\$ 75,000$ for funding through September 30, 1991,

Enclosed are two copies of the proposed interagency agreement. The agreement has been signed on behalf of DOE. If the agreement is acceptable to TVA, please sign the agreement on behalf of TVA and return one fully executed original to us.

Block 1.b. of the Face Page provides a space for TVA to assign an IA number. If TVA desires to assign an IA number, please indicate the number on the copy returned to us.

Any questions regarding this proposed agreement should be directed to Margie Henderson (FTS 626-0714) or commercial number (615) 576-0714.

ER-113:Henderson<smiles>[SiH3]C1CCCCC1</smiles>

William R. Bob

Assistant Manager for Defense Programs

Enclosures:

Interagency Agreement (2)

$S_{n, 1} \rightarrow P_{1} R$ 
CS-05 (REV 4-89)

REQUEST FOR OUTSIDE SERVICES CONTRACT (REIMBURSABLE)

Name of Contractor Department of Energy

street or P.O. Box Oak Ridge Operations, P.0. Box 2001

City Oak Ridge County Anderson State IN ZIP 37831 Phone (615) 576-0511

Contractor is a: $\mathrm{X}$ Federal Agency

State or Local Government Agency other

Wame and title of person signing contract for outside party Charles D. Crowe Contracting officer

TVA Program Manager Gregory W. Lowe Address Phone or Ext. 4455 (Responsible for this program)

Contract Administrators:

(Persons responsible for overseeing daily administration of contract)

TVA's Roger A. Milstead Address EB IA-K Phone or Ext. 6115

Contractor's Margie Henderson Address Same as Contractor Phone or Ext. 576-0714

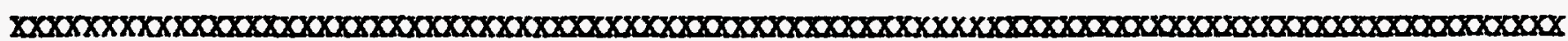
APPROVALS:

Level I Manager

Level II Manager

Level III Manager

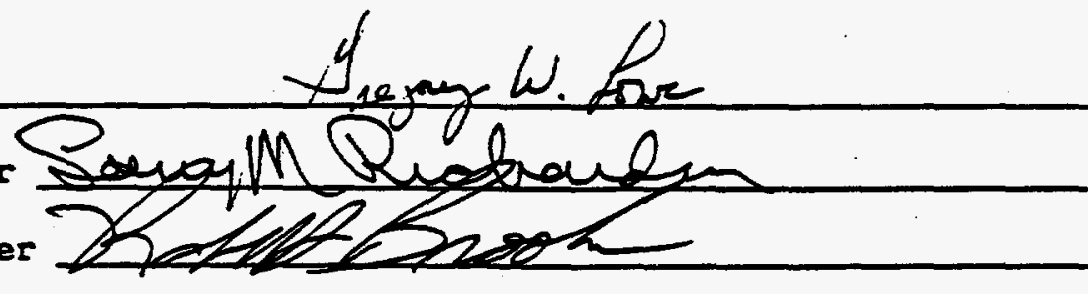
Date $5 / 22 / 91$ Date $5-2394$ Date 5232

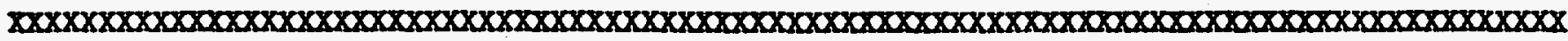
Date request received by Contract Services staff Request No. CS Type

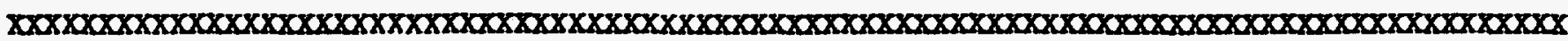

PLEASE SUBATT COLPLEIBD FORH, WIIH APPROPRIATE SIGNATURES, TO IHE CONIRACI SERVICES STAFE, OCH 2G 95B-K, FOR HANDLIYG. 
Effective date of contract 05/15/91

Termination date

$09 / 30 / 91$

Amount of contract $\$ 75,000.00$

Reimbursable account No. To be determined (If reimbursable contract number cannot be assigned prior to assignment of contract number, state "To be assigned."

Percentage of overhead to be applied against the contract

A11

(If percentage is less than applicable IVA overhead rate, justification memo is required from Level II Manager with concurrence from that organization's budget officer.)

Background Information: (Provide a brief summary of 'information and pertinent facts on this project and how it started. Why was IVA requested to perform the work? Attach copies of any pertinent correspondence, together with original and all copies of contract document when prepared by the other agency.)

IVA has contracted with the Department of Energy to perform flood hazard analyses at the Oak Ridge facilities for over 10 years. IVA has the technical expertise to perform these studies and a continuing interest in the development and use of up-to-date floodplain information.

IVA's Obligations: (Provide a detailed description of the program intent and goal and specific work to be performed by TVA.)

IVA will perform the requested study to provide updated flood hazard information at the oak Ridge facilities for siting of future development and use in IRC evaluation of existing structures.

Contractor's obligations: (Provide detailed description of Contractor's obligations in connection with the work to be performed by TVA.)

To provide funding for the study and any requested field data. 


$$
\text { 的, }
$$

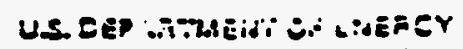

INTERAGENCY AGREEMENT FACE PAGE

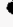

FunOS-OUT inTEMAGENCY AGREEMENT na!

newent te authority of Economy Act of 1932,

as amended (31. U.S.C. 1535); PL 95-91;

other:

2. TrRe of ACTION

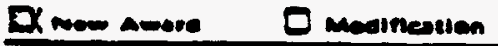

2. Daneer trtLedoesenimtion:

Flood Analyses in Support of Flood Emergency Planning (see pages 2-6)
a. DOE lA . Yo.:
b. Other ageney IA . Vo.:
c. HodiGeation No.: AOOO
d. Tack order No.:
e. pe No.
DE-AI05-910R21979
$05010 R 21070.000$

4. acneemert penioo (month. dey. yeor)

Fromi See Elock 9 Tor $9 / 30 / 91$

5. Finameial

2. Accounting and Appropriation Data:

$\begin{array}{lll}89 \times 0224.91 & \$ 40,800 & \text { (GB 03) } \\ \text { OR-14-91 } & \$ 21,400 & \text { (ORNL Overhead) } \\ 250 & \$ 12,800 \quad(\text { G\&A/GPS) } \\ 8202 & - & \end{array}$

b. Funding soures

Doe This Action

Aperey

Tores Funoum

c. Nieshod of Parmens:

Anome

X.

mesose

d. Amonat obiigated this action:

- 75,000

c. Invoicen, if any, mbmit to:

Danamine of Enitr.

P.9. $80 \times 2001$

Oak Ridge; IN 3783I-8622

c. PTIN: M. W. Henderson, ER-113

SF-10BO and cite thereon DE-AI05-910R21979

and Work Orders as stated in

Section IV Cost

9. renfonmune aeener neespravess

\section{(simarere)}

(date)

Yame (rypewritten):

Tille (rppewritzen):
6. DoE mogogan offierr

mama Danjel Hoag

Y-12 Environmenta 7 Safery, and

Health Branch, DP-813

mermat U.S. Department of Energy

P. 0. Box 2001

Oak Ridge, TN 37831

Tanomon numbers

(615) 576-0511 or FTS 626-0511

7. renFonmine ACENEY
2. Nermer
Tennessee Valley Authority
Evans Eldg. Room IW-141A
a. Acoremer
Knoxvilie, TN 37902-149g

Axeationa Dr. Ra Iph Brooks, Manager

Division of Water Resources

C. Program Director

Nanom

Acereare $7 . b$.

Tonemone $615-632-6770$

6. ISEUTHE ACENCY:

Depertenent of Enery (DOE)

Dak Ridge Operations Office

P.0. Box 2001

Oak Ridoe. TN 37837-8600

20. DoE Contranctine orficen:

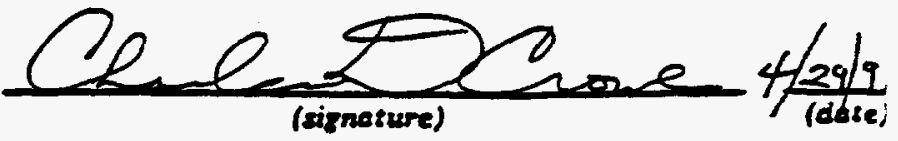

Diame (typesoritten): Charles D. Crowe

Contracting officer

Iitie (rypeuricten): Environmental Acquisitions

Procurement \& Contracts Div 
DOE Interagency Agreement No. DE-AI05-910R21979

Performing Agency: Tennessee Valley Authority

\section{Purpose}

Martin Marietta Energy Systems, Inc. (MMES), under contract to the U.S. Department of Energy (DOE), is in the process of performing natural phenomena, studies for the DOE's Y-12 Plant, Oak Ridge National Laboratory, and K-25 Site. The studies include flood analyses and development of flood emergency plans. DOE has a need for the technical support and assistance of the Tennessee Valley Authority (TVA) in providing the flood analyses. The dams involved are owned and operated by TVA, and TVA has extensive stream gage data on the Clinch River and associated tributaries.

II. Scope of Work

Task 1

a. Provide $\mathrm{Cl}$ inch River flood elevations in the vicinity of the Y-12 Plant for conditions resulting from the 25-year; 100-year; 500-year; 2,000-year; 10,000-year; 100,000-year; maximum probable; and probable maximum floods without the failure of Norris and Melton Hill Dams.

b. Provide Clinch River flood elevations in the vicinity of the Y-12 Plant for conditions resulting from the one-half probable maximum flood plus the failure of Norris Dam.

c. Provide Clinch River flood elevations in the vicinity of the $Y-12$ Plant for conditions resulting from failure of Norris Dam in non-flood conditions.

d. Provide Clinch River flood elevations for the flood conditions for (a.), (b.), and (c.) at the DOE pumping station located between $\mathrm{Cl}$ inch River miles 41 and 42 .

\section{Task 2}

Provide flood elevations for East Fork Poplar Creek from its mouth at Poplar Creek to the head of the stream for conditions resulting from the 25-year; 100-year; 500-year; 2,000-year; 10,000-year; 100,000-year; maximum probable; and probable maximum floods. Energy Systems will provide surveyed cross sections and pictures for locations as selected during discussions with the TVA. Also provide site drainage at five specific locations (Buildings 9201-2, 9201-3, 9204-1, 9720-1 and 9720-5) at the $Y-12$ Plant. 


\section{Scope of Work (cont'd)}

\section{Task 3}

Provide flood elevations for Bear Creek from its mouth at East Fork Poplar Creek to the head of the stream for the eight flood conditions as stated in Task 2. Energy Systems will provide surveyed cross sections for locations as selected during discussions with the TVA.

\section{Task 4}

Compute the local rainfall depth and duration data at the $Y-12$ Plant for conditions resulting from the 25-year; 100-year; 500-year; 2,000-year; 10,000-year; 100,000-year; maximum probable; and probable maximum precipitation.

\section{Task 5}

a. Provide $\mathrm{Cl}$ inch River flood elevations in the vicinity of the ORNL for conditions resulting from the 25-year; 100-year; 500-year; 2,000-year; 10,000-year; 100,000-year; maximum probable; and probable maximum floods without the failure of Norris and Melton Hill Dams.

b. Provide $\mathrm{Cl}$ inch River flood elevations in the vicinity of the ORNL for conditions resulting from the one-half probable maximum flood plus the failure of Norris Dam.

c. Provide Clinch River flood elevations in the vicinity of the ORNL for conditions resulting from failure of Norris Dam in non-flood conditions.

\section{Task 6}

Provide flood elevations for White 0ak Creek from its mouth at the Clinch River to the head of the stream for conditions resulting from the 25-year; 100-year; 500-year; 2,000-year; 10,000-year; 100,000-year; maximum probable; and probable maximum floods. Energy Systems will provide surveyed cross sections as required.

\section{Task 7}

Provide flood elevations for Melton Branch from its mouth at white Oak Creek to the head of the stream and for the tributary to Melton Branch for the eight flood conditions as stated in Task 6 . Energy Systems will provide surveyed cross sections as required.

\section{Task 8}

Compute the local rainfall depth and duration data at the ORNL for conditions resulting from the eight precipitation levels as listed in Task 4. 


\section{Scope of Work (cont'd)}

\section{Task 9}

a. Provide Clinch River flood elevations in the vicinity of the K-25 Site for conditions resulting from the 25-year; 100-year; 500-year; 2,000-year; 10,000-year; 100,000-year; maximum probable, and probabie maximum floods without the failure of Norris, Melton Hill, and Watts Bar Dams.

b. Provide Clinch River flood elevations in the vicinity of the K-25 Site for conditions resulting from the one-half probable maximum flood plus the failure of Norris Dam.

c. Provide Clinch River flood elevations in the vicinity of the K-25 Site for conditions resulting from the one-half probable maximum flood plus the failure of Melton Hill Dam.

d. Provide $\mathrm{Clinch}$ River flood elevations in the vicinity of the K-25 Site for.conditions resulting from failure of Norris Dam in non-flood conditions.

e. Provide $\mathrm{Cl}$ inch River flood elevations in the vicinity of the K-25 Site for conditions resulting from failure of Norris and Melton Hill Dam in non-flood conditions.

f. Provide Clinch River flood elevations for (a.), (b.), (c.), (d.), and (e.) at the two DOE pumping stations located between Clinch River miles 11 and 12, and 14 and 15.

Task 10

Provide flood elevations for Poplar Creek from its mouth at Watts Bar Lake to its junction with East Fork Poplar Creek for the eight flood conditions as stated in Task 6 . Energy Systems will provide surveyed cross sections as required.

Task 11

Compute the local rainfall depth and duration data at the K-25 Site for conditions resulting from the eight precipitation levels as stated in Task 4.

\section{Qual ity Assurance Requirements}

The calculations performed to support the tasks defined in the Scope of Work shall be prepared in accordance with ANSI/ASME NQA-1, 1986 Edition, or equivalent. The external interface between TVA and DOE as called for in the ANSI/ASME document will be handled by DOE. 
IV. Cost

The DOE hereby obligates $\$ 75,000$ to support this work through September 30,1991. The current obligation is based on the following cost estimates:

$\begin{array}{rrr}\text { Task } 1 & \$ 7,000 & \text { WO\# SO1080-16 } \\ \text { Task } 2 & 21,600 & \text { WO\# SO1080-16 } \\ \text { Task } 3 & 12,000 & \text { WO\# SO1080-16 } \\ \text { Task } 4 & 200 & \text { WO\# SO1080-16 } \\ \text { Task } 5 & 7,000 & \text { WO\# AK188GA1 } \\ \text { Task } 6 & 9,200 & \text { WO\# AK188GA1 } \\ \text { Task } 7 & 5,000 & \text { WO\# AK188GA1 } \\ \text { Task } 8 & 200 & \text { WO\# AK188GA1 } \\ \text { Task } 9 & 8,000 & \text { WO\# MUT310FP } \\ \text { Task 10 } & 4,600 & \text { WO\# MJT310FP } \\ \text { Task 11 } & 200 & \text { WO\# MJT310FP } \\ \text { TOTAL } & \$ 75,000 & \end{array}$

The cost for each task should be separately identified on the invoice and the appropriate work order indicated.

\section{v. Deliverables/Reports}

1. Clinch River flood elevations at the $Y-12$ P lant and DOE pumping station for the flood conditions as listed under Scope of Work for Task 1.

2. Tabulated water elevations for East Fork Poplar Creek during eight flood conditions as listed under Scope of Work for Task 2. Calculations and summary of analysis of flooding conditions at buildings and structures.

3. Tabulated water elevations on Bear Creek during eight flood conditions as listed under Scope of Work for Task 3.

Calculations and summary of analysis of flooding conditions.

4. Tabulated rainfall depth and duration data at the Y-12 Plant for eight precipitation levels as listed under Scope of Work for Task 4.

5. Clinch River flood elevations at the ORNL for the flood conditions as listed under Scope of Work for Task 5.

6. Tabulated water elevations for White Oak Creek during eight flood conditions as listed under Scope of Work for Task 6. Calculations and summary of analysis of flooding conditions at building and structures.

7. Tabulated water elevations for Melton Branch during eight flood conditions as listed under Scope of Work for Task 7. Calculations and summary of analysis of flooding conditions.

8. Tabulated rainfall depth and duration data at the ORNL for eight precipitation levels as listed under Scope of Work for Task 8. 
V. Deliverables/Reports (cont'd)

9. Clinch River flood elevations at the K-25 Site for the flood conditions as listed under Scope of Work for Task 9.

10. Tabulated water elevations for Poplar Creek during eight flood conditions as listed under Scope of Work for Task 10.

Calculations and summary of analysis of flooding conditions at buildings and structures.

11. Tabulated rainfall depth and duration data at the K-25 Site for the flood conditions as listed under Scope of Work for Task 11.

12. Final Report.

Deliverables shall be submitted to:

Manuel Duran

Martin Marietta Energy Systems, Inc.

P.0. Box 2009, Building 9739, Y-12 P1ant

Oak Ridge, Tennessee 37831-8209

VI. Duration of Agreement

See Block 4, Face Page.

VII. Program Officers

DOE and performing agency - see Block 6 and Block 7, Face

Page. Additional project representatives are as follows:

TVA - Gregory W. Lowe, Technical Contact (615) 632-4455

TVA - Janet L. Summers, Administrative Contact (615) 632-6440

MMES - R. 0. Daugherty, Technical Contact (615) 574-9638

MMES - Manuel Duran, Administrative Contact (615) 574-0705

DOE - Margie W. Henderson, Fiscal and Contractual Matters (615) 576-0714

\section{Obligation of Funds}

The total amount obligated by the U.S. Department of Energy is $\$ 75,000$.

IX. other

The attached Appendix A dated February 1982 is incorporated and made a part of this agreement. 


\author{
APPENDIX A \\ Standard General Provisions \\ For DOE-TVA Funds-out Interagency Agreements
}

I. Definitions. The term "Secretary" means the Secretary of Energy, the statutory head of the Department of Energy (hereinafter referred to as "DOE"), or any duly authorized representative thereof. The term "TVA" means the Tennessee Valley Authority, or any duly authorized representative of TVA. The term IA means Interagency Agreement.

II. Direct Costs Chargeable to DOE Funds. Direct costs are the costs that can be directly identified with and charged to the work under the agreement and within the limitations set forth below. Examples of such costs are salaries/wages, technical services, materials, travel and transportation, communications, and any facilities and equipment expressiy approved for purchase under the IA.
a. Expenditures for domestic travel expected to exceed $\$ 1,000$ per individual trip shall not be allowable hereunder without prior written approval of the DOE Contracting Officer.

b. Foreign travel costs are allowable only when the trip has received the advance approval of the DOE Contracting Officer.

c. Reimbursement for expenditures at technical meetings and seminars at which attendance is not required by DOE shall not be allowable without prior written approval of the DOE Contracting officer.

III. Financing: Reimbursement Agreement. Upon presentation by TVA of proper vouchers, the DOE shall reimburse TVA for costs actually incurred on DOE work subject to any limitations on costs otherwise provided in this agreement. Requests for reimbursement shall show separately the amounts to be reimbursed for (1) operating costs and (2) capital equipment (as defined in article entitled "Capital Equipment" hereof).

IV. Notice of Costs Approaching Total Estimated Costs. Whenever TVA has reason to believe that the total cost of the work under this agreement will be substantially greater or less than the presently estimated cost of the work, TVA shall promptly notify DOE in writing. TVA shall also notify DOE, in writing, when the aggregate of costs incurred and outstanding commitments allowable under the agreement is equal to 90 percent (or such other percentage as DOE may from time to time establish by notice to TVA) of the presently estimated total costs under this agreement. When the costs incurred and outstanding commitments equal 100 percent of such estimated total costs. TVA shall make no further commitments or expenditures (except to meet existing commitments) and shall be excused from further performance of the work unless and until DOE shall increase the total estimated costs to be incurred with respect to this agreement. 
V. Excess Funds. TVA shall take prompt action to return to DOE any funds determined to be excess to the work during the performance of the work and any unobligated funds after the completion of the agreement or as of September 30 each year uniess the agreement has been extended and any unused balances have been carried forward in the extension. In a joint venture project where TVA deposits the advance in any annual consolidated working fund, any unobligated balances shall be returned to DOE before the cutoff date at the close of each fiscal year.

VI. Financial Reports. TVA shall furnish DOE, not later than 15 days after the close of each reporting period, monthiy or other periodic cost or financial reports in such form and detail as may be required by DOE. Any costs incurred for capital equipment or other assets shall be supported by a list showing the description, make, any serial number and the cost of each item acquired.

VII. Accounting Records. TVA shall accumulate and account for obligations and costs incurred in connection with the work being performed under this agreement in such form and detail as may be required by DOE.

VIII. Termination. Either party may terminate this agreement upon 30 days written notice of such termination addressed to the other party. In the event of such termination, TVA shall be reimbursed for obligations actually incurred to the effective date of termination and for commitments extending beyond the effective date of termination to a date not later than the date upon which the agreement would have expired if not terminated under this paragraph, which TVA, in the exercise of due diligence, is unable to cancel. Payments under this agreement, including payments under this article, shall not exceed the ceiling amount elsewhere specified in this agreement.

IX. Capital Equipment.

a. "Capital Equipment" means each item of equipment which is expected to have an extended period of service, generally a year or more, and has sufficient monetary value, generally of $\$ 5000$ or more, to justify continuing accounting records for the item.

b. Unless expressly authorized by the Contracting officer in advance, TVA shall not be reimbursed or use funds made available under this agreement for the procurement or fabrication of capital equipment.

c. If capital equipment is purchased or otherwise acquired pursuant to an authorization under Paragraph (b) above, except as may be otherwise agreed by DOE and TVA:

(1) the title thereto shall vest in DOE;

(2) TVA shall be responsible for the maintenance and safeguarding 
(3) TVA shall maintain a record in such a manner as to assure adequate control and accounting satisfactory to DOE of capital equipment procured or fabricated.

$X$. Patents. It is understood and agreed by DOE and TVA that the rights to any invention or discovery made or conceived in the course of or under this agreement shall be determined as set forth below:

(1) Where the work is to be performed by a contractor or subcontractor of TVA, TVA agrees to incorporate the standard DOE Long Form Patent Rights clause of DOE PR 9-9.107-5 (a) except where the short form clause of DOE PR 9-9.107-6 is applicable. Paragraph ( $k$ ), Background Patents, of DOE PR 9-9.107-5 (a) should normally be deleted for contracts under $\$ 250,000$. Where, however, the contract or subcontract is with a small business firm or nonprofit organization, TVA shall use the attached patent rights clause entitled, "Patent Rights (Small Business Firms or Nonprofit Organizations)."

(2) Offerors and prospective contractors shall be provided with notice of and the opportunity to request, in accordance with applicable DOE regulations, at any time prior to the effective date of the contract or within 30 days thereafter, an advance waiver of all or any part of the rights of the United States with respect to practice in the course of or under the contract. The TVA Contracting office shall promptly forward such requests to DOE's patent counsel and will notify the contractor whether such request is granted in whole or in part.

(3) Where a contractor or subcontractor of TVA will not accept patent provisions provided by DOE, TVA shall not proceed with the contract or subcontract without written approval of DOE.

(4) Where the work performed results in an invention made or conceived by an employee of TVA, any such invention or discovery shall be reported and handled in accordance with the TVA Act and TVA patenting procedure. The rights to any such invention or discovery shall be determined by TVA. TVA agrees to grant to DOE an irrevocable, non-exclusive, paid-up license for the manufacture and use of such invention or discovery. DOE shall have the right to grant sublicenses for the use or manufacture of such invention or discovery.

(5) No claim for pecuniary award or compensation of any kind shall be asserted by TVA or its employees, consultants or contractors against DOE with reference to any such invention or discovery.

(6) It is recognized that during the course of the work under this agreement DOE or its employees, consultants, or contractors, may from time to time desire to publish, within the iimits of security requirements, information regarding scientific or technical developments made or conceived in the course of or under this agreement. In order that public disclosure of such information will not adversely affect the patent interests of DOE or TVA, patent approval for release and publication shall be secured from TVA prior to any such release or publication. 
XI. Technical Progress Reports: Publication. TVA will make such reports to DOE on the progress of the work under this agreement as may be mutually agreed upon.

It is the policy of DOE to make the results of the research, development and demonstration work contemplated under interagency agreements broadiy available to the scientific, technical and engineering comunity and others through the timely publication of reports or journal articles. All publications and engineering materials prepared under the IA will be freely exchanged, made available for public sales, unless classified, and a minimum of two copies sent to the DOE Technical Information Center (TIC), P. 0. Box 62, Oak Ridge, Tennessee 37830. Each IA technical report issued and each task order technical report issued pursuant to a master IA will be accompanied by a DOE Form 427 and a statement describing the technical reports delivered and will be sent to TIC for incorporation into the Technical Information Management System (TIMS).

XII. Environmental Safety and Health Requirements. DOE will not assume responsibility for prescribing and/or enforcing environmental safety and health requirements for operators of TVA facilities engaged in the performance of DOE work.

The following two articles are applicable if the work to be done involves classified information.

XIII. Security of Restricted Data.

a. It is TVA's duty to safeguard restricted data, formerly restricted data and other classified information. TVA shall, in accordance with DOE security regulations and requirements, be responsible for safeguarding restricted data, formerly restricted data and other classified information and protecting against sabotage, espionage, loss or theft of the classified documents and material in TVA's possession in connection with the performance of work under this agreement. Except as otherwise expressly provided in this agreement, TVA shall upon completion or termination of this agreement, transmit to DOE any classified matter in the possession of TVA or any person under TVA's control in connection with performance of this agreement. If retention by TVA of any classified matter is required after the completion or termination of the agreement and such retention is approved by DOE, TVA will complete a certificate of possession to be furnished to DOE specifying the classified matter to be retained. The certification shall identify the items and types or categories of matter retained, the conditions governing the retention of the matter and the period of retention, if known. If the retention is approved by DOE, the security provisions of the agreenent will continue to be applicable to the matter retained. 
b. Requlations. TVA agrees to conform to all security regulations and requirements of DOE.

c. Definition of Restricted Data. The term "restricted data", as used in this clause, means all data concerning: (1) design, manufacture or utilization of atomic weapons; (2) the production of special nuclear material; or (3) the use of special nuclear material in the production of energy, but shall not include data declassified or removed from the restricted data category pursuant to Section 142 of the Atomic Energy Act of 1954.

d. Definition of Formerly Restricted Data. The term "formerly restricted data", as used in this clause, means all data removed from the restricted data category under Section $142 \mathrm{~d}$ of the Atomic Energy Act of 1954, as amended.

e. Security Clearance of Personnel. TVA shall not permit any individual to have access to restricted data, formerly restricted data, or other classified information, except in accordance with the Atomic Energy Act of 1954, as amended, and DOE's regulations or requirements applicable to the particular type or category of classified information to which access is required.

f. Criminal Liability. It is understood that disclosures of restricted data, formerly restricted data or other classified matter relating to the work or services ordered hereunder to any restricted data, formerly restricted data or any other classified matter in connection with work under this agreement, may subject any representatives of TVA, its agents, employees or subcontractors to criminal liability under the laws of the United States. (See the Atomic Energy Act of 1954, as amended, 42 U.S.C. 2100 et seq.: 18 U.S.C. 793 and 794; and Executive Order 12065.)

g. Contracts and Purchase Orders. Except as otherwise authorized in writing by DOE, TVA shall insert provisions similar to the foregoing in all contracts and purchase orders under this agreement.

h. Security Requirements for Proprietary Energy Data. TVA shall safeguard DOE limited official use information, or other proprietary or sensitive data (including material relating to patents), from unauthorized access, disclosure, modification or destruction in accordance with applicable DOE security regulations and applicable orders and directives. TVA shall not have access to third party proprietary or business confidential information unless TVA shall have entered into a nondisclosure agreement with such third party. Any civil liability that TVA may have with respect to the disclosure or use of such information shall be governed by such nondisclosure agreement. 
i. Computer Security Requirements. In the event that this agreement involves utilization of a DOE computer system, TVA will establish administrative, technical and physical security procedures in accordance with applicable DOE regulations, to ensure against access to DOE information to individuals not formally authorized by DOE to possess such information. TVA shall not have access to third party proprietary or business confidential information uniess TVA shall have entered into a nondisclosure agreement with such third party. Any civil Tiability that TVA may have with respect to the disciosure or use of such information shall be governed by such nondisclosure agreement.

XIV. Classification. In the performance of the work under this agreement, TVA shall assign or obtain classifications to all documents, material and equipment originated or generated by TVA in accordance with classification guidance furnished to TVA by DOE. Every subcontract and purchase order issued hereunder involving the origination or generation of classified documents, material or equipment shall include a provision to the effect that in the performance of such subcontract or purchase order, the subcontractor or supplier shall assign classifications to all such documents, material and equipment in accordance with classification guidance furnished to such subcontractor or supplier by TVA.

XV. Real Property and Facilities:

TVA shall not be reimbursed or use funds made available under this agreement for the acquisition or condemnation of any real property or any facility or for plant or facility acquisition, construction or expansion. 
Attachment I

\section{PATENT RIGHTS}

(Small Business Firms or Nonprofit Organizations) (March 1982)

\section{a. Definitions}

(1) "Invention" means any invention or discovery which is or may be patentable or otherwise protectable under Title 35 of the United States Code (USC).

(2) "Subject Invention" means any invention of the contractor conceived or first actually reduced to practice in the performance of work under this contract.

(3) "Practical Application" means to manufacture in the case of a composition or product, to practice in the case of a process or method, or to operate in the case of a machine or system; and, in each case, under such conditions as to establish that the invention is utilized and that its benefits are, to the extent permitted by law or Government regulations, available to the public on reasonable terms.

(4) "Made" when used in relation to any invention means the conception or first actual reduction to practice of such invention.

(5) "Small Business Firm" means a small business concern as defined at Section 2 of Public Law 85-536 (15 USC 632) and implementing regulations of the Administrator of the Small Business Administration. For the purpose of this clause, the size standard for small business concerns involved in Government procurement, contained in 13 CFR 121.3-8, and in subcontracting, contained in 13 CFR 121.3-12, will be used.

(6) "Nonprofit Organization" means a university or other institution of higher education or an organization of the type described in section 501 (c) (3) of the Internal Revenue Code of 1954 (26 USC 501(c)) and exempt from taxation under section 501(a) of the Internal Revenue code (26 USC $501(a)$ ) or any nonprofit scientific or educational organization qualified under a state nonprofit organization statute.

(7) "Patent Counse1" means the Department of Energy (DOE) patent counsel assisting the DOE contracting activity.

\section{b. Allocation of Principal Rights}

The contractor may retain the entire right, title, and interest throughout the world to each subject invention subject to the provisions of this clause and 35 USC 203. With respect to any subject invention in which the contractor retains title, the Federal Government shall have a nonexclusive, nontransferable, irrevocable, paid-up license to practice or have practiced for or on behalf of the United States the subject invention throughout the world. 


\section{c. Invention Disclosure, Election of Title and Filing of Patent Applications by Contractor}

(1) The contractor will disclose each subject invention to the Patent Counsel (with notification by the Patent Counsel to the Contracting officer) within two months after the inventor discloses it in writing to contractor personnel responsible for the Administration of patent matters. The disclosure to the Patent Counsel shall be in the form of a written report and shall identify the contract under which the invention was made and the inventor(s). It shall be sufficiently complete in technical detail to convey a clear understanding, to the extent known at the time of the disclosure, of the nature, purpose, operation, and the physical, chemical, biological or electrical characteristics of the invention. The disclosure shall also identify any publication, on sale or public use of the invention and whether a manuscript describing the invention has been submitted for publication and, if so, whether it has been accepted for publication at the time of disciosure. In addition, after disclosure to the Patent Counsel, the contractor will promptiy notify the Patent Counsel of the acceptance of any manuscript describing the invention or of any on sale or public use planned by the contractor.

(2) The contractor will elect in writing whether or not to retain title to any such invention by notifying the Patent Counsel within twelve months of disclosure to the contractor; provided that in any case where publication, on sale or public use has initiated the one year statutory period wherein valid patent protection can still be obtained in the United States, the period for election of title terminates sixty days prior to the end of the statutory period.

(3) The contractor will file its initial patent application on an elected invention within two years after election or, if earlier, prior to the end of any statutory period wherein valid patent protection can be obtained in the United States after a publication, on sale, or public use. The contractor will file patent applications in additional countries within either ten months of the corresponding initial patent application or six months from the date permission is granted by the Commissioner of Patents and Trademarks to file foreign patent applications where such filing has been prohitited by a Secrecy order.

(4) Requests for extension of the time for disclosure to the Patent Counsel, election, and filing, may, at the discretion of the Patent Counsel be granted.

\section{d. Conditions When the Government May Obtain Title}

(1) The contractor will convey to DOE, upon written request, title to any subject invention: 
(i) If the contractor fails to disclose or elect the subject invention within the times specified in (c) above, or elects not to retain title.

(ii) In those countries in which the contractor fails to file patent applications within the times specified in (c) above; provided, however, that if the contractor has filed a patent application in a country after the times specified in (c) above but prior to its receipt of the written request of the Patent Counsel, the contractor shall continue to retain title in that country; or

(iii) In any country in which the contractor decides not to continue the prosecution of any application for, to pay the maintenance fees on, or defend in a reexamination or opposition proceeding on, a patent on a subject invention.

\section{e. Minimum Rights to Contractor}

(1) The contractor will retain a nonexclusive, royalty-free license throughout the world. in each subject invention to which the Government obtains title except if the contractor fails to disclose the subject invention within the times specified in (c) above. The contractor's license extends to its domestic subsidiaries and affiliates, if any, within the corporate structure of which the contractor is a part and includes the right to grant sublicenses of the same scope to the extent the contractor was legally obligated to do so at the time the contract was awarded. The license is transferable only with the approval of DOE except when transferred to the successor of that part of the contractor's business to which the invention pertains.

(2) The contractor's domestic license may be revoked or modified by DOE to the extent necessary to achieve expeditious practical application of the subject invention pursuant to an application for an exclusive license submitted in accordance with 10 CFR 781. This license will not be revoked in that field of use or the geographical areas in which the contractor has achieved practical application and continues to make the benefits of the invention reasonably accessible to the public. The license in any foreign country may be revoked or modified at the discretion of DOE to the extent the contractor, its licensees, or its domestic subsidiaries or affiliates have failed to achieve practical application in that foreign country.

(3) Before revocation or modification of the license, DOE will furnish the contractor a written notice of its intention to revoke or modify the license, and the contractor will be allowed thirty days (or such other time as may be authorized by DOE for good cause shown by the contractor) after the notice to show cause why the ficense should not be revoked or modified. The contractor has the right to appeal, in accordance with 10 CFR 781, any decision concerning the revocation or modification of its license. 
f. Contractor Action to Protect Government's Interest

(1) The contractor agrees to execute or to have executed and promptly deliver to the Patent Counsel all instruments necessary to:

(i) Establish or confirm the rights the Government has throughout the world in those subject inventions for which the contractor retains title, and

(ii) Convey title to DOE when requested under (d) above and to enable the Government to obtain patent protection throughout the world in that subject invention.

(2) The contractor agrees to require, by written agreement, its employees, other than clerical and nontechnical employees, to disclose promptly in writing to personnel identified as responsible for the administration of patent matters and in a format suggested by the contractor each subject invention made under this contract in order that the contractor can comply with the disclosure provisions of (c) above and to execute all papers necessary to file patent applications on subject inventions. The disclosure format should require, as a minimum, the information requested by (c)(1) above. The contractor shall instruct such employees through the empioyee agreements or suitable educational programs on the importance of reporting inventions in sufficient time to permit the filing of patent applications prior to United States or foreign statutory bars.

(3) The contractor will notify the Patent Counsel of any decision not to continue prosecution of a patent application, pay maintenance fees, or defend in a reexamination or opposition proceeding on a patent, in any country, not less than thirty days before the expiration of the response period required by the relevant patent office.

(4) The contractor agrees to include, within the specification of any United States patent application and any patent issuing thereon covering a subject invention, the following statement, "This invention was made with Government support under (identify the contract) awarded by the Department of Energy. The Government has certain rights in this invention."

(5) The contractor agrees to:

(i) Provide a report prior to the close-out of the contract listing all subject inventions;

(ii) Provide notification of all subcontracts under this contract for experimental, development, demonstration, or research work, the identity of the patent rights clause therein, and copy of each subcontract upon request;

(iii) Provide promptly a copy of the patent application, filing date, serial number, patent number and issue date for any subject invention in any country in which the contractor has applied for a patent. 


\section{g. Subcontracts}

(1) The contractor will include this clause, suitably modified to identify the parties, in all subcontracts, regardless of tier, for experimental, developmental or research work to be performed by a small business firm or a domestic nonprofit organization. The subcontractor will retain all rights provided for the contractor in this clause, and the contractor will not, as part of the consideration for awarding the subcontract, obtain rights in the subcontractor's subject inventions.

(2) The contractor will include in all other subcontracts, regardless of tier, for experimental, developmental, demonstration, or research work the patent rights clause required by 41 CFR 9-9.107-5(a) or 41 CFR 9-9.107-6 as appropriate, modified to identify the parties.

(3) In the case of a subcontract at any tier, DOE the subcontractor, and the contractor agree that the mutual obligations of the parties created by this clause constitute a contract between the subcontractor and DOE with respect to those matters covered by this clause.

\section{h. Reporting on Utilization of Subject Inventions}

The contractor agrees to submit on request periodic reports no more frequentiy than annualiy on the utilization of a subject invention or on efforts at obtaining such utilization that are being made by the contractor or its licensees or assignees. Such reports shall include information regarding the status of development, date of first commercial sale or use, gross royalties received by the contractor, and such other data and information as DOE may reasonably specify. The contractor also agrees to provide additional reports as may be requested by DOE in connection with any march-in proceeding undertaken by DOE in accordance with paragraph $(j)$ of this clause. To the extent data or information supplied under this section is considered by the contractor, its licensee or assignee to be privileged and confidential and is so marked, DOE agrees that, to the extent permitted by 35 USC $202(c)$ (5), it will not disclose such information to persons outside the Government.

\section{i. Preference for United States Industry}

Notwithstariding any other provision of this clause, the contractor agrees that neither it nor any assignee will grant to any person the exclusive right to use or sell any subject invention in the United States unless such person agrees that any products embodying the subject invention or produced through the use of the subject invention will be manufactured substantially in the United States. However, in individual cases, the requirement for such any agreement may be waived by DOE upon a showing by the contractor or its assignee that reasonable but unsuccessful efforts have been made to grant licenses on similar terms to potential licensees that would be likely to manufacture substantially in the United States or that under the circumstances domestic manufacture is not comercially feasible. 


\section{j. March-in Rights}

The contractor agrees that with respect to any subject invention in which it has acquired title, DOE has the right in accordance with the procedures in OMB Circular A-124 to require the contractor, an assignee or exclusive licensee of a subject invention to grant a nonexclusive, partially exclusive, or exclusive license in any field of use to a responsible applicant or applicants, upon terms that are reasonable under the circumstances, and if the contractor, assignee, or exclusive licensee refuses such a request, DOE has the right to grant such a license itself if DOE determines that:

(1) Such action is necessary because the contractor or assignee has not taken, or is not expected to take within a reasonable time, effective steps to achieve practical application of the subject invention in such field of use;

(2) Such action is necessary to alleviate health or safety needs which are not reasonably satisfied by the contractor, assignee, or licensees; or

(4) Such actions is necessary because the agreement required by (i) of this clause has not been obtained or waived or because a licensee of the exclusive right to use or sell any subject invention in the United States is in breach of such agreement.

k. Special Provisions for Contracts with Nonprofit Organizations If the contractor is a non-profit organization, it agrees that:

(1) Rights to a subject invention in the United States may not be assigned without the approval of DOE, except where such assignment is made to an organization which has as one of its primary functions the management of inventions and which is not, itself, engaged in or does not hold a substantial interest in other organizations engaged in the manufacture or sale of products or the use of processes that might utilize the invention or be in competition with embodiments of the invention (provided that such assignee will be subject to the same provisions as the contractor);

(2) The contractor may not grant exclusive licenses under United States patents or patent applications in subject inventions to persons other than small business firms for a period in excess of the earlier of: 
invention; or

(i) five years from first commercial sale or use of the

(ii) eight years from the date of the exclusive license excepting that time before regulatory agencies necessary to obtain premarket clearance, unless on a case-by-case basis, DOE approves a longer exclusive license. If exclusive field of use licenses are granted, commercial sale or use in one field of use will not be deemed commercial sale or use as to other fields of use, and a first commercial sale or use with respect to a product of the invention will not be deemed to end the exclusive period to different subsequent products covered by the invention;

(3) The contractor will share royalties collected on a subject invention with the inventor; and

(4) The balance of any royalties or income earned by the contractor with respect to subject inventions, after payment of expenses (including payments to inventors) incidental to the administration of subject inventions, will be utilized for the support of scientific research or education.

\title{
1. Communications
}

The DOE central point of contact for communications or matters relating to this clause is the Patent Counsel.

\author{
END OF CLAUSE
}




\section{REEEREANCES}

1. Garrison, J. M., Granju, J. P., and Price, J. T., "Unsteady Flow Simulation in Rivers and Reservoirs," Journal of the Hydraulics Division, ASCE, Volume 95, No. HY5, Proceedings Paper 6771, September 1969, pp. 1559-1576.

2. U.S. Water Resources Council, Guidelines for Determining Flood Elow Erequency, Bulletin No. 17B of the Bydrology Subcomittee, Washington, D.C., September 1981.

3. U.S. Geological Survey, Technique for Estimating Magnitude and Erequency of Floods in Tennessee, 1976.

4. U.S. Geological Survey, Magnitude and Frequency of Urban Floods in the United States, August 1981.

5. U.S. Department of Commerce, Weather Bureau, Technical Paper No. 40 , Rainfall Erequencr Atlas of the United States for Durations From 30 Minutes to 24 Hours and Return Periods Erom 1 to 100 Years, D. M. Hershfield, Washington, D.C., May 1961.

6. Newton, Donald W., and Vinyard, J. W., "Computer-Determined Unit Hydrographs From Floods," Journal of the Bydraulics Division, ASCE, Volume 93, No. HY5, September 1967.

7. Final Safety Analysis Report, Watts Bar Nuclear Plant, Docket Nos. 50-390, 50-391.

8. National Weather Service, Probable Maximum and IVA Precipitation Estimates With Areal Distribution for Tennessee River Drainares Iess Than 3.000 Mi. ${ }^{2}$ in Area, Hydrometeorological Report No. 56, October 1986.

9. U.S. Weather Bureau, Probable Maximum and IVA Precipitation Over the Tennessee River Basin Above Chattanoera, Hydrometeorological Report No. 41, 1965.

10. U.S. Army Corps of Engineers, Hydrologic Engineering Center, HEC-2 Water Surface Profiles, Computer Prooram 723-X6-L202A, Davis, California, Version 4.6.0; February 1991.

11. Tschantr, B. A., Thite Oak Creek Hydrologic and Spiliway Adequacy Analysis, report (Document No. ORNL/SUB/87-32-CI213V-1) submitted to Martin Marietta Energy Systems, Inc., August 1987.

12. Preliminary Safety Analysis Report, Clinch River Breeder Reactor Plant, Volume 1, Project Management Corporation.

13. National Oceanic and Atmospheric Administration, National Weather Service, Five- to 60-Minute Precipitation Frequency for the Eastern and Central United States, NOAA Technical Memorandum NWS HYDRO-35, June 1977. 
14. Newton, D. W., and Cripe, M. W., "Flood Studies for Safety of TVA Nuclear Plants, Hydrologic and Embaniment Breaching Analysis," Tennessee Valley Authority, Knoxrille, Tennessee, 1973.

15. Newton, Donald W., "Improving Probable Maximum Flood Estimates," paper presented at the Symposium on Surface-Water Impoundments, Minneapolis, Minnestota, June 1980.

16. National Weather Service, Probable Maximum and TVA Precipitation for Tennessee River Basins up to 3.000 square Miles in Area and Durations to 72 Bours, Bydrometeorological Report No. 45, 1969, with Addendum of Jume 1973:

17. National Weather Service, Comparison of Generalized Estimates of Probable Maximum Precipitation With Greatest Observed Rainfalls, NOAA Technical Report NWS 25, March 1980.

18. Gumbel, E. J., Statistical Theory of Extreme Values and Some Practical Apolications, National Bureau of Standards, Applied Mathematic Series 33, February 1954.

19. American Nuclear Society, Determining Design Basis Flooding at Power Reactor Sites, ANSI/ANS 2.8, 1981. 


\title{
INTERNAL DISTRIBUTION
}

\author{
T. A. Angelelli \\ J. W. Baker \\ W. W. Bowman \\ W. R. Brock \\ W. K. Crowley \\ D. R. Denton \\ J. O. Evans \\ K. E. Fricke \\ J. D. Huggins \\ R. J. Kroon \\ W. E. Manrod III \\ C. T. McLoughlin \\ W. E. Miller \\ W. J. Moore \\ T. J. Pierce \\ D. G. Renfro \\ T. L. Ryan \\ K. E. Shaffer \\ B. K. Williams \\ W. R. Williams \\ File-RJH-RC \\ Central Files-RC (2) \\ CNPE Library (5)
}

\section{EXTERNAL DISTRIBUTION}

Office of Scientific and Technical Information (2), U. S. Department of Energy, Oak Ridge Field Office

Project Manager

U. S. Department of Energy, Oak Ridge Field Office 